\title{
PREDICTIONS OF A NON-GAUSSIAN MODEL FOR LARGE SCALE STRUCTURE
}

\author{
Z. H. Fan \& J. M. Bardeen
}

Department of Physics. F:M-15

The University of Vashington

Seattle. Mashington 9819.5

June 26. 1992

\section{PREPARED FOR THE U.S. DEPARTMENT OF ENERGY}

This report was prepared as an account of work sponsored by the United States Government. Neither the United States nor the United States Department of Eneigy, nor any of their employees, nor any of their contractors, subcontractors, or their employees, makes any warranty, express or implied, or assumes any legal liability or responsibility for the product or process disclosed, or represents that its use would not infringe privately-owned rights. By acceptance of this article, the publisher and/or recipient acknowledges the U.S. Government's right to retain a nonexclusive. royalty-free license in and to any copyright covering this paper. 
A modified CDMI model for the origin of structure in the universe. based on an inflation model with two interacting scalar fields, is analyzed to make predictions for the statistical properties of the density and velocity fields and the microwave background anisotropy. The initial gauge-invariant potential $\zeta$ which is defined as $\zeta=\delta \rho /(\rho+p)+3 \varphi$, where $\psi$ is the curvature perturbation amplitude and $p$ is the pressure, is the sum of a Gaussian field $O_{1}$ and the square of a Gaussian field $\phi_{2}$. We assume a Harrison-Zel'dovich scale-invariant power spectrum for $\phi_{1}$, and a log-normal 'peak' power spectrum for $o_{2}$. The location and the width of the peak are described by parameters $k_{c}$ and $a$. respectively. Comparing our theoretical calculations for two dimensional density angular correlation function to the results of the APM survey, we set certain parameter regime for $k_{c}, a$ and the amplitude ratio of Gaussian and non-Gaussian fields. The model is motivated to some extent by inflation models with two interacting scalar fields, but is mainly interesting as an example of a model whose statistical properties change with scale. On small scales, it is almost identical to a standard scale-invariant Gaussian CDM model. On scales near the location of the peak of the non-Gaussian field, the distributions have long tails in high positive values of the density and velocity fields. Thus, it is easier to get large-scale streaming velocities than the standard CDM model. We calculate the quadrupole amplitude of fluctuations of the cosmic microwave background radiation and the rms variation of the temperature field smoothed with a $10^{\circ} \mathrm{FWH.M}$ Gaussian. and find a reasonable agreement with the new COBE results.

Biasing should be important in our model. We discuss one of the possible biasing schemes very briefy, and further discussions on this will be in our future work.

Subject headings: large scale structure - - statistics -- cosmic background radiation - cosmology - galaxies: clustering 


\section{INTRODLCTION}

The formation of large scale structure is an important subject in cosmology: Among the various models attempting to explain large scale structures. the cold dark matter (CDM) model is one of the most successful models. It has three basic assumptions:

(1). The initial perturbations arise from quantum fuctuations during inflation and are statistically Gaussian random fields with a scale-invariant power spectrum (Bardeen, Steinhardt, \& Turner 1983).

(2). The perturbations evolve in a universe with approximately $90 \%$ of the non-relativistic matter in the form of weakly interacting particles with negligible velocity dispersion.

(3). The cosmological density parameter for the present universe is dominated by the non-relativistic matter and is $\Omega=1$. as expected from inflation.

For a Gaussian field, all statistical properties can be described by two-point correlation function $\xi(\vec{x})$, or the power spectrum which as defined by Salopek et al. $(1959)$ is

$$
P(k)=\frac{k^{3}}{2 \pi^{2}} \int d^{3} x \exp (-i \vec{k} \cdot \vec{x}) \xi(\vec{x})
$$

the power per unit logarithmic interval in $k$.

The initial perturbation field is best expressed in terms of the gauge-invariant potential $\zeta$

$$
\dot{\zeta}=\dot{\varphi} p \cdot(p+p)+3 \vec{r}
$$

where $p$ is the pressure, and $\varphi$ is the curvature perturbation amplitude. (Bardeen et al. 1986 hereafter BBIS). Scale-inariance means that the primordial $\zeta$. eraluated when all astrophysically relevant scales have wavelength larger than the Hubble radius, has a power spectrum with roughly the same amplitude on each logarithmic interval in wave number $\dot{h} . P(k) \approx$ constant.

The standard CDM model wor:s quite well at small scales if the Hubble constant $H_{0} \approx 50 \mathrm{~km} \mathrm{sec}{ }^{-1} \mathrm{Mpc}^{-1}$ (Daris et al. 1955). but seems less successful on scales larger than $10 \mathrm{Mpch}^{-1}$ i $:$ here $h$ is the Hubble constant in units of 
$100 \mathrm{~km} \mathrm{sec}^{-1} \mathrm{Mpc}^{-1}$ ). (White et al. 10Si: Efstathion. Sutherland. \& Maddox 1990). Two recent statistically well-founded pieces of evidence are the galaxygalaxy angular correlations from the AP.M suriey: and the QDOT redshift survey ( Maddox et al. 1990, Saunders et al. 1991. Efstathiou et al. 1990). This means that we need some extra power on those scales comparing to the prediction of the standard CD.M model.

There are models which have completely different mechitnisms from CDM to explain the large scale structure (Hogan 1991: Hill. Schramm. \& Fry 1989; Ostriker \& Cowie 1981; Turok 1989). Still in the framework of (D.M, there are some ways to modify the standard model. Efstathiou et al. (1990) consider a non-zero cosmological constant model. Bond et al.(1991) consider the effect of an unstable $17 \mathrm{kev}$ massive neutrino, and propose a mechanism which increases the neutrino species' parameter $\theta$. The basic idea in these two models is to get larger characteristic scale $\lambda_{e q} \approx 2 \pi \times 3.7 \theta^{1 / 2} /(\Omega h)\left(\mathrm{Mpch}^{-1}\right)$. which is the comoving horizon scale at matter and radiation equality (BBLS). The other way is to drop out the assumption of a scale-invariant power spectrum (Salopek et al. 1089. Kiofman et al. 1959), but still assume a Gaussian field. There are more parameters to be tuned in this kind of models to fit the observational results. There is another way which gives up the Gaussian assumption. Pcebles (1983) is the first to point out that it is possible to get some non-Gaussian fuctuations by non-linear interactions. and they may have some interesting effects on large scale structures. A lot of work has been done on generating non-Gaussian fields (Wise 1985; Salopek 1991; Tofman et al. 19S9: Ostriter \&: Cowie 19S1: Turok 19S9: La \& Steinhardt 19S9). Recently Matarrese et al. (1090.1001a.1991b. hereafter MML.M). and Weinberg and Cole (1992. Preprint, hereafter $\mathrm{IV}^{\circ} \mathrm{C}$ ) have done simulations on various kinds of non-Gaussian models. They found that the skewness of the density perturbation field is an important parameter to classify different kinds of non-Gaussian models. The general conclusion is that skew-positive models produce more high density clumpy structures than Gaussian models which have zero skewness. while skew-negative models give more void structures. (MML.M: MC). MMLM favor skew-negative models arguing the they have bubble-like structures which agree with the observations (Geller (t Huck:a 1959). But the quantitative 
calculations show that skew-negative muciels give too many roids comparing to the observations while skew-positive mode!s agree with observations on topological properties better (IVC). IVC also pointed out the disadrantages of shew-positive models, e.g., they need very strong biasing to create empty roids. In MMLM papers, they consider a scale-invariant power spectrum, and in $\mathrm{IVC}$. a power law spectrum is considered.

In this paper, we present a model which is basically a skew-positive model. Our model thus shares some general properties of the latter. For example, there are more high density clumpy structures. and the formation of quasars and galaxies occurs earlier than that predicted by the standard CDM. But we have two important differences from MIML.M and IVC. One is that in ou model. we assume the initial perturbation field is a combination of a Gaussian and a non-Gaussian field rather than a purely non-Gaussian field. Motivated by a particular class of double inflation models (Salopek. Bond. S Bardeen 1959, Bardeen 1992), the gauge-invariant potential $\zeta$ is assumed to be a combination of a Gaussian and the square of a Gaussian field

$$
\zeta=0,+0 ?
$$

where $O_{1}$ and $\phi_{2}$ are two independent Gaussian felds. The other difference is that we use a non-scale invariant power spectrum fo: $O_{2}$. which is

$$
\left.P_{\phi_{2}}(k)=B^{1 / 2} \exp \{-2 a ! \ln (k \cdot k \cdot))^{2}\right\}
$$

where $a$. and $k_{c}$ are parameters which represent the width and the location of the peak of the power spectrum. and $B^{1 / 2}$ is the amplitude of the non-Gaussian field. For $\dot{o}_{1}$, we assume the Harrison-Zel dorich spectrum: $P_{\theta_{1}}(k)=A$. where $A$ is a constant.

The statistical properties change with scale: On small scales. Gaussian statistics are dominant. but near characteristic scale related to $k_{c}$ the non-Gaussian contributions are important.

We use the same normalization condition as $\mathrm{II}^{\circ} \mathrm{C}$ to normalize the total amplitude of density perturbation field to 1 when averaged over a sphere of $S \mathrm{Mpch}^{-1}$. (WC: Daris \& Peebles 10S3). Then the ratio of the Gaussian and the nonGaussian fields $A / B$ is another adjusiable parameter. 
This paper mainly concentrates on using the linear theory to compare our model with the standard CD.M by discussing statistical properties and two-point correlation functions which are directly related to the power spectrum. We assume $h=0.5$ and $\Omega=1$, as conventional for C'D.M, though direct observations of $h$ are increasingly convergent toward a value of 0.8 .

The paper is organized as following: In Sec.2. we discuss the distribution function of the total density field. and show how it changes with different smoothing scales, different characteristic scales as characterized by $k_{c}$, and different ratios $A / B$. In Sec.3, by comparing the theoretical calculations with the results of the APM (the Automatic Plate Measuring, Maddox et al. 1990) survey, we set an acceptable parameter regime for $a, k_{.}$. and $A / B$. In Sec. 4 . the properties of velocity fields are discussed. In Sec.j. we consider the fluctuations on the cosmic microwave background radiation. The quadrupole term and the rms rariation smoothed with $\theta_{F W H M}=10^{\circ}$ Gaussian are calculated. They are compared to the new Cosmic Background Explorer (COBE) results, and we find that our model agree with the COBE results reasonably.. In Sec.6. Several realizations are presented to compare the visual impres : ons with the standard CDM model. In Sec.T. we make some discussions and comments on our work. 


\section{THE DISTRIBUTION FUNCTION OF THE DENSITY FIELD}

In this section, we consider the distribution function of the density perturbation field which is a combination of a Gaussian and a non-Gaussian field.

The power spectrum of linear density perturbations is related to that of the initial gauge-invariant potential $\zeta$ through a transfer function $T(k)$

$$
P_{\underline{\underline{B}}}(k)=T^{2}(k) P_{\zeta}(k)
$$

For CDM models, detailed calculations give (Bond \& Efstathiou 1984)

$$
T\left(K^{*}\right) \times k^{2} /\left\{1+\left[a k+(b k)^{3 / 2}+(c k)^{2}\right]^{\nu}\right\}^{1 / \nu}
$$

where $a=6.4\left(\Omega h^{2}\right)^{-1}$ Mpc. $b=3.0\left(\Omega h^{2}\right)^{-1}$ Mpc. $c=1 . i\left(\Omega h^{2}\right)^{-1} \mathrm{Mpc}$ and $\nu=$ 1.13. For $k$ much smaller than $k_{: 4}=2 \pi / \lambda_{2 q} . T(k) \times k^{2}$. Transforming to the spatial coordinates gives $\dot{\delta} \rho / \rho \times-r^{-2} \zeta$. We use this approximation to discuss the qualitative statistical properties of the density perturbation fields, but for the normalization based on $\left\langle(\delta \rho / \rho)^{2}\right\rangle$ averaged over a sphere of $S \mathrm{Mpch}^{-1}$, the full transfer function (2.2) is used.

With $\zeta=0_{1}+\left(O_{2}\right)^{2}$. we have

$$
\operatorname{cop} / p-\Gamma^{2} 0_{1}-r^{2} \cdot 2
$$

where $O_{1}$ and $O_{2}$ are two independent Gaussian Selcis. Their power spectra are given in the last section. $a . k_{c}$ and $f / B$ are parameters which are adjustable in our model. We normalize $\delta \rho / \rho$ to 1 when averaged over a sphere with a radius $8 \mathrm{Mpch}^{-1}$. There is no any bias in this paper.

Write $\delta \rho / \rho$ as

$$
\delta \rho / \rho=\sigma_{i i^{2} L_{G}}+2 \sigma_{\hat{v} 2} \sigma_{22} y
$$

where $\nu_{G}$ is the dimensionless Gaussian part. and $y$ is the dimensionless nonGaussian part. $\sigma_{0 G}, \sigma_{02}$ and $\sigma_{2 ?}$ are defined as

$$
\begin{aligned}
& \sigma_{0 G}^{2}=\int d \ln k \quad P_{u}(\dot{i}) \\
& \sigma_{02}^{2}=\int d \ln k P_{\partial_{2}}(\therefore)
\end{aligned}
$$




$$
\sigma_{? 2}^{2}=j d \ln k k^{4} P_{0,}(k)
$$

where $P_{G}$ stands for the power s!)ectrum of the Gaussian density perturbations.

We need to consider the joint distribution function for $\nu_{G}$ and $y$. Since they are independent of each other. the joint distribution is:

$$
P\left(\nu_{G} \cdot y\right) d \nu_{G} \dot{\therefore} y=P\left(\nu_{G}\right) P(y) d \nu_{G} d y
$$

where $P\left(\nu_{G}\right)$ is simply a Gaussian distribution

$$
P\left(\nu_{G}\right)=\frac{1}{(2 \pi)^{1 / 2}} \exp \left(-\nu_{G}^{2} / 2\right)
$$

and $P(y)$ is obtained from the joint distribution function of $o_{2}, \nabla \phi_{2}$, and $\nabla^{2} \phi_{2}$

$$
\begin{aligned}
P(y)= & \frac{4 \pi}{(2 \pi)^{5 / 2}\left(1-\gamma_{2}^{2}\right)^{1 / 2}} \int d y_{0} d l \cdot r^{2}|| y_{0} \mid \\
& \exp \left[-\frac{y_{0}^{2}}{2\left(1-\gamma_{2}^{2}\right)}-\frac{\left(y+i_{2} r^{2} / 3\right)^{2}}{2 y_{0}^{2}\left(1-i_{2}^{2}\right)}+\frac{i 2\left(y+i_{2} r^{2} / 3\right)}{1-i_{2}^{2}}-(1 / 2) r^{2}\right]
\end{aligned}
$$

where $\gamma_{2}=\sigma_{12}^{2} /\left(\sigma_{02} \sigma_{22}\right)$, and $\sigma_{12}^{2}=j d \ln h k{ }^{2} P_{02}(k)$.

Let $\nu$ be the total dimensionless iensity perturbation. i.e. $\nu=\sigma_{0}^{-1} \delta \rho / \rho$, where $\sigma_{0}^{2}$ is $\left\langle(\delta \rho / \rho)^{2}\right\rangle$. Then

$$
\nu_{G}=\left(\sigma_{i}:-2 \tau_{32} \sigma_{22} y\right) / \sigma_{0 G}
$$

Replace $\nu_{G}$ in eq. (2.9) by eq. (2.1:1. aisd integrate over $y$ to get the distribution function for $\nu$,

$$
\left.P(\nu) d \nu=\left\{\int \exp \left[-\frac{\sigma_{0} \nu-2 \sigma_{02} \sigma_{22} y}{\sqrt{2} \sigma_{0 G}}\right)^{2}\right] P(y) d y\right\} d \nu
$$

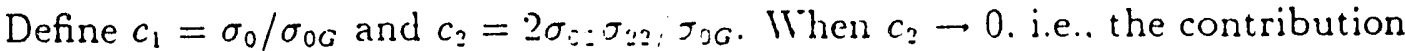
of the non-Gaussian part goes to zero. (2.12) becomes a Gaussian distribution. On the other hand when $c_{1} \rightarrow x$. 2.12) tends to have the form of (2.10) with $y=(\delta \rho / \rho) /\left(2 \sigma_{02} \sigma_{22}\right)$.

The behavior of the distribution (2.12) depends on $a . k_{c}$, and $A / B$. If we consider a smoothed field. it also depencis on the smoothing scale $R_{f}$. We use a Gaussian smoothing function. 
In Fig.1, we plot the purely non-Gaussian distribution $P(y)$ with different $; 2$. They are skew-positive, and have higher probabilities at high $\sigma$-level perturbations than that of the Gaussian distribution. The smaller the $\gamma_{2}$ is. the sharper peaked at zero the distribution is. and it gets broader when 12 goes to 1 .

Fig.2 shows the behavior of $P\left(\nu^{\prime}\right)$ with clifferent $k_{\varphi}, A / B$, and $R_{\ell}$. When the smoothing scale $R_{\mathrm{f}}$ is small. the Gaussian field is dominant. and the distribution is nearly symmetric. For $R_{f}=10 \mathrm{Mpch}^{-1}$. and $R_{f}=20 \mathrm{Mpch}^{-1}$, : he distribution function is no longer symmetric. and has a long tail at high positive values, which is the general property of a skew-positive model related to the earlier formation of quasars and galaxies than that of the standard CDM. The larger the $A / B$ is , the more Gaussian the distribution tends to be. Note that for $R_{f}=2 \mathrm{Mpch}^{-1}$, our approximation (2.3) is not ralid. But the tendency described above is true.

Comparing with observations, the standard C'D.M model works quite well on small scales $r<10 \mathrm{Mpch}^{-1}$. but on scales larger than $10 \mathrm{Mpch}^{-1}$. more power than predicted by the standard CDM model is needed to explain the strong correlations on those scales. (Davis \& Peebles 19\$3: Maddox et al 1990) One of the adrantages of our model is that the statistical properties of perturbation fields depend on scale, and on small scales. Gaussian fields are dominant, thus keeps the advantages of Gaussian fields ( $\left.\mathrm{H}^{\circ} \mathrm{C}^{\prime}\right)$. By choosing parameters $a, k$, and $A / B$ properly, we can get large correlations on scales larger than $10 \mathrm{Mpch}^{-1}$. In next section, we calculate the angular correlation function of density perturbations, and compare it with the APM survey to get an acceptable parameter regime. 


\section{THE LIMITATIONS ON PARAMETERS}

Using the APMI technique. Maddox et al (1990) got more reliable results than those of the Lick survey (Groth $\&$. Peebles $19 T i$ ) on the two dimensional correlation function $w(\theta)$ shown in Fig.3. The dotted line is the result of the standard CDMI model with $\Omega=1$ and $h=0.5$. It is clearly seen that there is insufficient power on large scales.

The angular correlation function is related to the spatial two-point correlation function by an integral equation (Peebles 10S0). Here we use $\Omega=1$, which corresponds to $q_{0}=0.5$. The same selection function as in Maddox et al (1990) is used in our calculations.

In our model, the power spectrum for the total density field is

$$
P(k)=T^{2}(k)\left[P_{0,1}(k)+P_{s_{3}^{2}}(k)\right]
$$

where $T(k)$ is the transfer function given by $\{2.2$.

$P_{\phi_{1}}(k)=A$ is used. $P_{\partial_{2}^{2}}(k)$ is related to the power spectrum of $O_{2}$ by the following equation:

$$
P_{0 ;}(k)=\left.\frac{k^{3}}{2 \pi^{2}} j \frac{d^{3} \vec{k}_{i}}{(2 \pi)^{3}} 0_{21} \vec{k}_{1} l^{3} s_{2}\left(\vec{k}-\vec{k}_{1}\right)\right|^{2}
$$

We calculate $u(\theta)$ for different $a . k \ldots$ and.$-1 / B$. It is known that from quantum fluctuation theories (Salopek et al. 1959), ve can zot get a rery sharp peak in the power spectrum for $\mathrm{O}_{2}$. Thus we restrict a to be less than 1 . Calculations show that:

(1). For $k_{e q} / k_{c}<5$, the curve of $u\langle\theta\rangle$ is alwa: $s$ too steep to match the APM results, which means that the characteristic sca:e related to $k$, is too small to account for the extra power on large scales needed by the observational results.

(2). For $k_{e q} / k_{c}>14$. the curve is always too $\therefore$ at. i.e.. the characteristic scale is too large.

(3). In the range $j \leq k_{\text {eq }} / k \leq 1 \div$. we can find proper $a$ and $A / B$ to fit the $A P M$ results. In Table.1. we ':st the proper ange for $a . k$, and $A / B$. For 
example, when $k_{e q} / k_{0}=10 . a=0 . \bar{v}$. then $A / B$ should be in the range $0.0165 \leq$ $A / B \leq 0.0302$. The two dimensional correlation functions $u(\theta)$ for $k_{e q} / k_{c}=10$, $a=0.5$ are shown in Fig.t along with the results calculated using the standard CDM model.

Bardeen, Bond St Efstathiou (19ST) discussed what kind of a power spectrum of the peculiar velocity field is needed to match the observations. They concluded that it is better to have a spectrum with a wider peak shifted to a larger scale than the predictions of the standard CD.M model. In Fig.j. we plot the power spectra of the total velocity field of our model with a group of parameters and that of the standard CDM model. We do get a wider peak which shifts to a larger scale. Calculations on 3-dim. rms velocity for different smoothing scales are listed in Table.2. We see. the rms velocities of our model are larger than those predicted by the standard CD.M model. On small scales they seem to be too large. But here we do not consider any biasing. For a skew-positive model like ours, the biasing should be stronger than that of the standard CDM model (WC 1991) because the density distribution is less homogenous. and the probabilities for large positive density perturbations are higher than those of the Gaussian case. The strong biasing will reduce the rms peculiar velocities. At the same time it will also reduce the quadrupole perturbation $a_{2}$ of the microware background radiation, and make $a_{2}$ be too small. Decreasing a. i.e. flattening the peak of the power spectrum will increase $a_{2}$. but the power on small scales will be increased as well since our power spectrum of non-Gaussian field is logarithmically symmetric respect to $k_{c}$. One possible way to get rid of this problem is to make the peak asymmetric, and put more power on large scales. An appropriately designed effective scalar field potential in inflation can accomplish this. 


\section{STATISTICAL ANALYSIS ON THE PECULIAR VELOCITY FIELD}

In this section, we discuss the correlations and the distribution function of the peculiar velocity field using the linear theory. and compare them with those of the standard CD.I model.

First, we consider the correlation of $3-$ a:m $\because$ ocity $\vec{\imath}(\vec{x})$ :

$$
\left.\xi_{2 b}(r)=\langle\vec{r}(\vec{r}) \cdot \vec{r} \cdot \vec{x}-\vec{r})\right\rangle
$$

This quantity gives us information on the bulk velocity field. A positive $\xi_{1 b}(r)$ means that on average particles separated by $r$ move coherently toward the same direction.

Using the linear theory $\xi_{10}(r)$ is related to the power spectrum of the initial perturbation field by

$$
\xi_{u b}(r)=\int d \ln k T^{2}(k) / k^{2} P_{b}(\Leftrightarrow) \sin (k r) /(k r)
$$

where $P_{6}(k)$ stands for the initial power spectrum of the gauge-invariant potential $\zeta . T(k)$ is the transfer function of $(2.2)$.

We calculate $\xi_{10}(r)$ for several groups of parameters as shown in Fig.6. On comving scales less than the characteristic scale. the correlations are larger than those of the standard CDM model. This is cons:stent with the physical picture. In our model. there are more dense regions arourd the characteristic scale. and on scales less than that. more pairs of particles move toward the same dense clump, thus we get larger $\xi_{2 b}$. For points $:$ hose separatica is larger than the typical scale of clumps, they have higher probabilities to move in the way either falling to the same clump from opposite directions or falling to different clumps. Both of them tend to reduce $\xi_{1 b}$.

Now we consider the correlation of $\vec{\imath}(\vec{x}) \cdot \hat{x}$ :

$$
\xi_{u}(r)=-\langle(\vec{v}(\vec{r}) \cdot \hat{r} \cdot \vec{v}(\vec{x}-\vec{r}) \cdot \hat{r})\rangle
$$

$\xi_{\text {ui }}(r)$ represents the property of the infall reocit $\because$. The minus sign in (4.3) means that we define the correlation to be positive :f $t \cdots$ points move toward each other. 
$\xi_{u}(r)$ is related to the power spectrum by:

$$
\xi_{v i}(r)=\int d \ln k T^{2}(k) / k^{2} P_{i}(k)\left[\frac{\sin (k r)}{(k r)}+\frac{2 \cos (k r)}{(k \cdot r)^{2}}-\frac{2 \sin (k r)}{(k r)^{3}}\right\}
$$

Fig. 7 shows $\xi_{i i}(r)$. On certain scales. our model gives larger correlations, and the coherent scale is larger than that of the standard CD.I model. This is again consistent with that our r:odel is skew-pusitive and has more clumpy structures.

By analysing the corretitions of the streaming velocity and the infall velocity, it shows that our model can have larger coherent motion and the coherent scales than those predicted by the stanciard CDM model.

Furthermore, we calculate the rms velocity of $\vec{v}(\vec{x}, R)$, the average velocity over certain scales $R$.

$$
\vec{r}(\vec{x} \cdot R) \equiv \frac{1}{1} \int_{\mid \vec{r} \leq R} d^{3} \vec{r} \vec{r}(\vec{x}+\vec{r})
$$

where $V$ is the rolume of the sphere with a radius $R$.

The rms value reflects how strong the motion is coherent on scales less than $R$. And the rms of $\vec{v}(\vec{x}, R)$ can also be calculated from the power spectrum:

$$
<\vec{v}(\vec{x} \cdot R) \cdot \vec{v}(\vec{x} \cdot R)>=\int d \ln k \cdot T^{2}(k) / k^{2} P_{i}(k) \mu r_{i}^{2}(k \cdot R)
$$

where

$$
\Pi_{i}(k \cdot R)=\frac{3\{-k \cdot R \cos i k R)+\sin (k R)]}{(k R)^{3}} .
$$

Fig. S shows the behaviors of the rms values. We see that for the same scale which is less than the characteristic scale. our model has larger ms velocity than that of the standard CDM model. In another word. we can get larger regions in which the motion is coherent. Some obserrations (Dressler 19S9) show that there is a very large coinerent flow. $\sim 600 \mathrm{kms}^{-1}$. extending at least $30 \mathrm{Mpch}^{-1}$. It is very hard to get such large coherent motion over such large scales in the standard CDM model. While in our model. we can have such large coherent motions over 20-30 $\mathrm{Mpch}^{-1}$. This is again because we have dense structures over large scales in our model, which are more like 'Great Attractors'.

Another quantity we calculate is the :ms of $v_{r}(\vec{x}, R)$. Here $v_{r}(\vec{x}, R)$ is defined as

$$
v_{r}(\vec{x} \cdot R) \equiv \frac{1}{\Gamma} \int_{i \vec{r} \leq R} d^{3} \vec{r} \vec{r}(\vec{x}+\vec{r}) \cdot \hat{r}
$$


The rms of $\operatorname{rr}(\vec{x}, R)$ is related to the power spectrum by

$$
<l_{r}^{2}(\vec{x} . R)>=\int d \ln k \quad T^{2}(k) / k^{2} P_{i}(k) H H_{2}^{2}(k R)
$$

and

$$
W_{2}(k R)=\frac{3\left[k \cdot R \sin (k \cdot R)-4 \sin ^{2}(k R / 2)\right]}{(k \cdot R)^{3}} .
$$

This rms ralue shows that how strong the gravitation is and what a large region it can affect. This can be seen from the Virial theorem: $v \sim(G M / R)^{1 / 2}$, where $M$ is the mass in the sphere. Since we have more large and dense structures, we expect to get larger rms of $v_{r}$.

The results are ploted in Fig.9. we do see there are larger values than those of the standard CDM model on scales less than the characteristic scale.

The analysis above is mainly related to the power spectrum. To understand the non-Gaussian perperties further. we need to calculate the distribution function of the velocity field.

Write the total velocity as

$$
\vec{r}_{\text {total }}=\vec{r}_{G}+\vec{r}_{G G}
$$

where $\vec{r}_{G}$ stands for the Gaussian part of the relocity field, and $\vec{v}_{v G}$ is the nonGaussian part.

Lsing the linear theory: we have

$$
\begin{aligned}
& \vec{r}_{i}=\Gamma O_{1} . \\
& \vec{v}_{V G}=\Gamma O_{2}^{2} .
\end{aligned}
$$

Let us first consider the distribution of the purely non-Gaussian part $\tilde{i}_{\text {. }}$. Since $\phi_{2}$ is a Gaussian field. $\Gamma_{1} o_{3}(i=1,2.3)$ is also a Gaussian field. Because $\left\langle\mathrm{O}_{2} \nabla_{i} O_{2}\right\rangle=0$, the joint probability of $O_{2}$ and $\nabla_{1} O_{2}$ is simply:

$$
\begin{aligned}
P\left(\dot{O}_{2}, \eta_{1}, \eta_{2} . \eta_{3}\right) d O_{2} d \eta_{1} d \eta_{2} d \eta_{3}= & \left(\frac{1}{2 \pi}\right)^{2} \frac{1}{\sigma_{12}}\left(\frac{3_{1 / 2}^{1 / 2}}{\sigma_{! 2}}\right)^{3} \exp \left(-\frac{O_{2}^{2}}{2 \sigma_{02}^{2}}\right) \\
& \exp \left[-\frac{3\left(\eta_{1}^{2}-\eta_{2}^{2}+\eta_{3}^{2}\right)}{2 \sigma_{12}^{2}}\right] d O_{2} d \eta_{1} d \eta_{2} d \eta_{3}
\end{aligned}
$$


With $\nu_{2}=0_{2} / \sigma_{02}$ and $\eta^{2}=\eta_{1}^{2}+\eta_{2}^{2}+\eta_{3}^{2} \cdot(4.10)$ turns out to be

$$
P\left(\nu_{2}, \eta\right) d \nu_{2} d \eta=\frac{1}{(2 \pi)^{1 / 2}}\left(\frac{5 t}{\pi}\right)^{1 / 2} \frac{1}{\sigma_{: 2}^{3}} \exp \left(-\nu_{2}^{2} / 2\right) \exp \left[-3 \eta^{2} /\left(2 \sigma_{12}^{2}\right)\right] \eta^{2} d \nu d \eta
$$

where $\sigma_{02}$ and $\sigma_{12}$ are defined as before.

Use $\vec{v}_{N G}=2 \mathrm{O}_{2} \nabla \mathrm{O}_{2}$, we can find the distribution function for $v \cdot N G$. It is

$$
\begin{aligned}
& P\left(v_{\text {VG }}\right) d v_{Y G}=\frac{1}{(2 \pi)^{1 / 2}}\left(\frac{j \frac{1}{\pi}}{\pi} j^{1 / 2} \frac{1}{\sigma_{12}^{3}} \frac{v^{2} \cdot G}{\Sigma \sigma_{02}^{3}}\right. \\
& \int_{-\infty}^{\infty} \exp \left(-\nu_{2}^{2} / 2\right) \exp \left(-3 v_{1 G G}^{2} i\left(S \sigma_{02}^{2} \sigma_{12}^{2} \nu_{2}^{2}\right) !\left|\nu_{2}^{-3}\right| d \nu_{2} d v_{N G}\right.
\end{aligned}
$$

With $x=v_{N G} /\left(2 \sigma_{02} \sigma_{12}\right)$, the distribution function for $x$ is

$$
P(x) d x=\frac{1}{(2 \pi)^{1 / 2}}\left(\frac{54}{\pi}\right)^{1 / 2} x^{2} \int_{-x}^{x} \exp \left(-1_{2}^{2} / 2\right) \exp \left[-3 x^{2} /\left(2 \nu_{2}^{2}\right)\right]\left|\nu_{2}^{-3}\right| d \nu_{2} d x
$$

We see there is no parameter in it. and the distribution for $x$ is unique and independent of $a$ and $k$. This is diarent from the density field which distribution depends on the parameter $: 3$.

In Fig.9, we show the behavio:s of $P(x)$ along with the distribution function for the Gaussian velocity field. We see they are quite different. The non-Gaussian distribution has non-zero probabil:ty at $x=0$. and a long tail at high $x$ values.

Now we consider the distribution for the total velocity. Since $\vec{r}_{G}$ and $\vec{r}_{. G}$ are independent of each other. the join: distribution is the product of the distribution functions for $v_{i,}$ and for $\left.v_{i, j}, i=1.2 .3\right)$ :

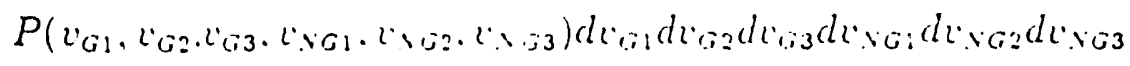

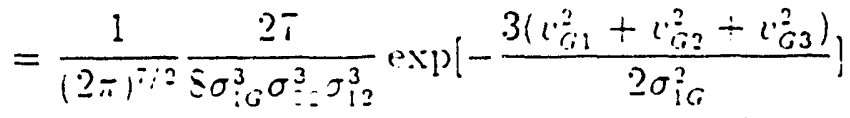

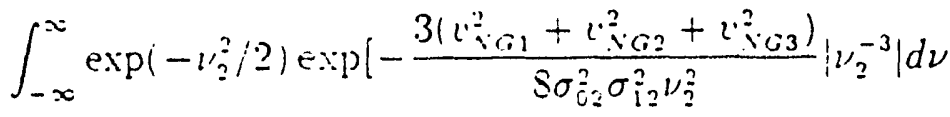

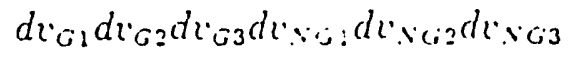

where $\sigma_{1 G}$ is the rms of $\left.i_{G}, \sigma_{1 G}=<l_{G}^{2}\right\rangle^{1 / 2}$.

Use $v_{i}=v_{G i}+v_{\text {. }} G_{i}(i=1,2.3)$ as variables. we get

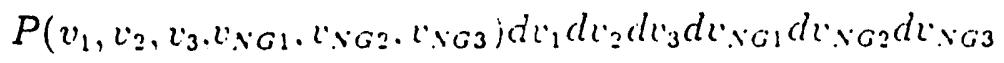

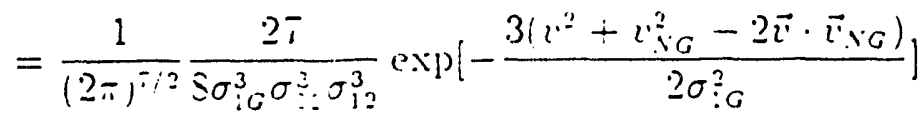

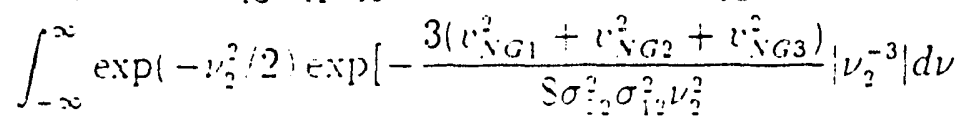

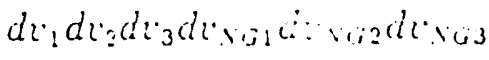


Define $b_{1}=\sigma_{02} \sigma_{12} / \sigma_{1 G}$ and $b_{2}=\sigma_{11} \sigma_{: G}$. where $\sigma_{11}$ is defined as $<r^{2}>^{1 / 2}$. Integrate over all other variables except $r_{1}^{\prime}=r_{1} / \sigma_{11}$. we get (hereafer we still use $v$ for $v^{\prime}$ )

$$
p(v) d v=\frac{54}{(2 \pi)^{3 / 2}} \frac{b_{2}^{2}}{\left(6 b_{1}\right)} \cdot \exp \left(-3 b_{2}^{2} v^{2} / 2\right) f(v) d v
$$

where

$$
\begin{aligned}
f(v)=\int_{-\infty}^{\infty} d \nu \int_{0}^{\infty} d x & x_{1}^{\prime}\left|\nu_{2}^{-3}\right| \exp \left(-\nu_{2}^{2} / 2\right) \exp \left[-3 x^{2} /\left(2 \nu_{2}^{2}\right)\right] \\
& \exp \left(-6 b_{1}^{2} x^{2}\right)\left[\exp \left(6 b_{1} b_{2} x v^{*}\right)-\exp \left(-6 b_{1} b_{2} x v^{\cdot}\right)\right]
\end{aligned}
$$

The distribution $P(v)$ depends on $b_{1}$ and $b_{2}$ which are functions of $a . k_{c}$ and $A / B$. As $b_{1} \rightarrow 0$, i.e., the contribution from the non-Gaussian field goes to zero, the distribution (4.17) tends to the distribution for a Gaussian velocity field which is

$$
P\left(v^{2}\right) d v^{\prime}=\left(\frac{54}{\pi}\right)^{1 / 2} v^{2} \exp \left(-3 v^{2} / 2\right) d v
$$

When $b_{1} \rightarrow \infty$, i.e., the Gaussian part goes to zero. (4.17) goes to (4.13).

Fig.10 shows $P\left({ }^{\prime \prime}\right)$ for sereral groups of parameters. We see that for the smoothing scale equal to $2 \mathrm{Mpch}^{-1}$. the distribution is very near the distribution for a purely Gaussian velocity field. as it is for larger smoothing scales when $A / B$ is larger. When the smoothing scale is near $k_{-}^{-i}$ and $A / B$ is smaller, the distribution has a long tail in high velocities. Thus as the density ficld. the statistical properties of the velocity field changes with scales.

Thus there are two effects make it eavier to get high velocities on certain scales than using the standard CDM mociel. Cne is from our particular choice of the power spectrum for the non-Gaussian field. and this gives higher rms velocities. The other effect is purely statistical. We get a long tail in high velocities which means that we have higher probabilities to get high relocities than the standard CDM model.

One thing needs to be mentioned. Our analysis in this paper is for the mass field, and we do not take into account any biasing effect. Since our model. like all other skew-positive models. las larger probabilites for high positive density perturbations. the biasing effect should be important. In the last section. we put forward a possible biasing scheme for our model. more detailed work will be done in our future work. 


\section{FLUCTUATIONS OF COSMIC MICROWAVE BACKGROUND RADIATION}

Fluctuations of the cosmic microware background radiation (CMBR) provide us the most restrictive constrains to theoretical models. Recent reports from the COBE show that the rms quadrupole amplitude of temperature fluctuations is $13 \pm 4 \mu \mathrm{K}$. and the rms sk! variation. smoothed with a $10^{\circ}$ FIVHM Gaussian. is $31 \pm \tau_{\iota} \mathrm{K}$.

In this section, we calculate the rms quadrupole amplitude and the rms variation of the temperature field using our combination model, and it shows that our model can agree with the COBE results quite well.

The multipole amplitude is defined as (Bond \& Efstathiou 198T)

$$
\Delta T_{i}^{2}=\frac{1}{4 \pi} \sum_{m=-1}^{m=+1}\left|a_{i m}\right|^{2}
$$

and $\left.a_{i}^{2} \equiv<\left|a_{1 m}\right|^{2}\right\rangle$ is related to the power spectrum of the gauge-invariant initial potential $\zeta$ as (Peebles 19S?)

$$
a_{i}^{2}=\pi H^{4} \int_{0}^{\infty} \frac{d k}{h} P_{i}(k) j_{i}^{2}\left(k \tau_{0}\right)
$$

where $\tau \equiv \int d t / a$ is the proper time. and the subscripte zero stands for the present value.

The two point correlation function of the smouthed temperature field is ( $\mathrm{Wi}$ son \& Silk 10S1)

$$
C\left(\theta, \theta_{3}\right)=\int_{0}^{2} C^{\prime}(0) R\left(0 . \theta \cdot \theta_{3}\right) 0 d 0
$$

where $\theta_{s}=0.425 \theta_{F W H . M}$ is the Gaussian snoothing angle.

$$
R\left(0, \theta, \theta_{s}\right)=\frac{1}{2 \theta_{3}^{2}} \exp \left(-\frac{\theta^{2}+0^{2}}{4 \theta_{3}^{2}}\right) I_{0}\left(\frac{\theta_{0}}{2 \theta_{3}^{2}}\right) \text {. }
$$

and $C(\varphi)$ is the unsmoothed two point correlation function. After substracting the mean and dipole term. it is (Górshi 1991)

$$
C(0)=\frac{1}{4} H^{4} \int_{0}^{\infty} \frac{d k}{k} P_{i}(k)\left[j_{i}\left(k \tau_{0} 0\right)-j_{0}^{2}\left(k \tau_{0}\right)-3 j_{i}^{2}\left(k \tau_{0}\right)\left(1-\frac{0^{2}}{2}\right)\right] .
$$

The rms variation $\sigma\left(\theta_{0}\right)$ is defined as (Bond (- Efstathiou 19ST)

$$
\sigma\left(\dot{\theta}_{s}\right) \equiv[C(0, \theta, 1)]^{\frac{1}{2}}
$$


Integrating over $\phi$, we get the runs variation is

$$
\sigma^{2}\left(\theta_{s}\right)=\frac{1}{4} H^{4} \int_{0}^{\infty} \frac{d k}{k} P_{\zeta}(k)\left[F\left(1: \frac{3}{2}:-\left(k \tau_{i} \theta_{s}\right)^{2}\right)-j_{0}^{2}\left(k \tau_{n}\right)-3 j_{i}^{2}\left(k \tau_{0}\right)\left(1-2 \theta_{s}^{2}\right) !\right. \text {. }
$$

where $F(\alpha ; \gamma ; z)$ is the confluent hypergeometric function. and

$$
F\left(1 ; \frac{3}{2} ;-\left(k \tau_{0} \theta_{s}\right)^{2}\right)=\int_{0}^{1} \exp \left[\left(k \tau_{0} \theta_{s}\right)^{2}\left(t^{2}-1\right)\right] d t
$$

The results are listed in Table.3. For the parameters we use. the main contributions to $(\Delta T / T)_{2}$ and $\sigma\left(\theta_{F W H M}=10^{\circ}\right)$ come from the Gaussian field. For $(\Delta T / T)_{2}^{2}$, the non-Gaussian contribution is only $\sim 1.7 \%$ of the Gaussian contribution. For $\sigma^{2}\left(\theta_{F W H M}=10^{\circ}\right)$, it is $\sim 14 . i \%$.

It is known that the quadrupole fluctuations are the results of very large scale perturbations, and large $\theta_{F w} m . M$ correspond to large scales. too. We use a 'peak' power spectrum for the non-Gaussian field. thus it is important only on certain scales which depend on $k_{c}$ and $a$. For the $k_{r}$ and $a$ we choose here, they are smaller than those scales which contribute to $(\Delta T / T)_{2}$ and $\sigma\left(\theta_{F W H M}=10^{\circ}\right)$. Recluce $a$ and $k_{c}$, the contributions will be larger. The contribution to $\sigma$ from non. Gaussian field depends on $\theta_{F W H . M}$. Fig.12 shows the contributions to $\sigma^{2}$ from the Gaussian field and from the non-Gaussian feld relative to $\theta_{F W H M}$. When $\theta_{F W H M}>3^{\circ}$. the Gaussian part is larger. White for $\theta_{F W H} \times 3^{\circ}$. the non-Gaussian part is dominant.

Compare with the C'OBE results on the quaclupole term and the rms variation with $\theta_{F W H M}=10^{\circ}$ (Smoot et al. 1992). We find that our model can fit both the angular correlation function of the clensity perturbation field and the C.MBR fluctuations reasonably well. 


\section{MIORPHOLOGY}

In this section, we use the adhesion model (Weinberg d Gunn 1990: Shandarin \& Zel'dovich 19S9: Shandarin 19Ss: Kofman. Pogosyan S: Shanarin 1990) to construct several realizations of the velocity fields both for the standard CDM model and for our model. Because of the limitation in the dynamic range for doing realizations, we use $a=0.5$, and $k_{i}=k_{,} / 5$ for the non-Gaussian field.

Fig. 13(a) and 13(b) are slices of the velocity fiolds for the standard CDM model and our combinational model using the linear theory. It can be seen that in our model, there are some large clumps, while in the standard CDM model, it is more random and homogeneous on such large scales. The large coherent structures in our model make the correlations on large scales larger than those in the standard CD.I model. Fig. $14(a)$ and $14(b)$ correspond to Fig.13(a) and 13(b) respectively, but some non-linear effects are taken into account by using the adhesion model with the 'finite $\nu$ ' method (Weinberg et al. 1990: Shandarin 1988). We see that the patterns are almost the same as linear realizations (Fig.11). but some small scale structures are smoothed out. (Neinberg et al 1000)

We have two different ways of analysing statistical properties including nonlinear evolution effects in the framework of the adhesion model (Shandarin 19S8). One is the simulation method with finite $\nu$ (Nusser \& Dekel 1090: Weinberg et al 1990), the other is the 'slicleton method' in the limit of $\nu$ going to zero (Kofman et al 1990). Future work will apply these adhesion model techniques more extensively. 


\section{DISCLSSION}

We discuss a model in which the initial perturbation field $\zeta$ is a combination of a Gaussian and a square of a Gaussian field with some particular power spectra. There are three parameters in this model being adjusted: $a . k_{c}$ and $A / B$. By comparing with the AP.I survey, we get certain acceptable ranges for them. Then we compare our model with the standard CD.M model.

Our model is basically a skew-positive model. and there are more large scale clumpy structures and fewer roids than the standard CD.I model (see Fig.13(b) and $14(\mathrm{~b})$ ) if we assume that galaxy traces mass. This seems to be inconsistant with observations. Thus some biasing mechanisms are needed in our model. Since it is a skew-positive, we expect the existence of a strong biasing. WC pointed out that with ccrtain biasing, the skew-positive models can agree with the observations best on topological properties whereas both Gaussian and skew-negative models have too many roids. For our model. we use peak' power spectrum for the non-Gaussian field, and the characteristic scale is much larger than the typical galaxy scale. Thus one possible biasing scheme is still to follow the basic idea of 'peak' biasing (BBIS 10S6; Kaiser 19S5). but assume that the non-Gaussian field only changes the large scale 'background' field. and has no contributions to the number of peaks with galaxy scales. High density regions over certain large scales enhances the number of peaks of the Gatussian part. which are higher than a certain threshold (BBIS 19S6). Me will consider this farther in subsequent papers.

Our theoretical motiration is based on a specific double inflation model, but our model is an interesting example of certain kind of models whose statistical properties change with scale. On small scales. it is basically Gaussian dominant, thus the adrantages of Gaussian models on small scale still exist. By adjusting parameters properly; we can fit both the AP.I two-dimensional correlations and the $C O B E$ results quite well. Analysis of the peculiar velocity ficlds shows that on scales less than the characteristic scale related to $k$, the rms bulk velocities as well as the correlations of the bulk velocity are larger than those of the standard CDM model. Further more the non-Gaussian field have a long tail at high positive values 
for the velocity distribution. These two effects make it easier to get large bulk velocities than the standard CDMI model. On scales larger than the characteristic scale, the rms bulk velocites become less than those predicted by the standard CDM. This is because we have dense and large clumps in our model, and on scales less than the typical scale of clumps. matter mainly moves toward the same clump, and gives larger bulk velocities. While on scales larger than the typical scale, matter moves mainly falling either into the same clump from opposite sides or into different clumps. This makes the bulk relocities smaller. Our model seems to be more attractive if 'Great Attractors' really exist in the universe. We also analyse the average infall velocity orer a sphere with certain radius $R$. The peak of the rms velocity distribution with $R$ is higher and broader than the standard CDM model, and the peak shifts to larger length scales depending on the characteristic scale we choose for the non-Gaussian field. This again shows that our model is more consistant with a 'Great Attractor' model than standard CDM.

The calculations on the fuctuations of the cosmic microware background radiation show that with $\Omega=1$, and $h=0.5$. our model can agree with the results of both the AP.I survey and the COBE.

Couchman et al. (1902) made some simulations with the standard CDM model trying to trace galaxies when they cluster. and they claimed that there is a significant bias in the relocities of galaxies relative to the dark matter. Thus they suggest that by considering the anti-bias. the standard CD.I model might be consistent with the observations of large bulk peculiar velocity. We do not consider this effect. What we do here is to use the same normalization condition $\left(\sigma_{8}=1\right)$ for both the standard CD.I model and for our model to compare them and look for the differences between these two models.

The ability to fit observational clata on a range of scales cannot be considered a success of the model, in that the model has plenty of adjustable parameters. However it is interesting to explore some of the ways non-standard statistics can affect comparison with observations.

While this work is mainly based on linear pertubation theory, we plan to use the adhesion model to gain insight into the non-linear evolution of large scale 
structure in these models.

Acknowledgements

This research was supported in part by the Department of Energy under Grant o. DE-FG06 91ER40614. 


\section{REFERENCES}

Bardeen, J. .1. 19S8, in The Second Guo Shou-jing Summer School on Particle Physics and Cosmology, ed. A. Zee (Gordon and Breach, London. 1988)

Bardeen, J. M., Bond, J. R.. Kaiser. .‥ d Szalay A. S. 19S6. ApJ, 304, 15 (BBKS)

Bardeen, J. M., Bond, J. R.. S. Efstathiou. G. 19ST, ApJ, 321, 2S

Bardeen, J. M., Steinhardt. P. J.. d Tuner. M. S. 19S3. Phys. Rev. D., 28, 679

Bardeen, J. M. 1992, in preparation

Bond, J. R. 1991. Phys. Lett. B.. 265. 245

Bond, J. R.. \& Efstathiou. G. 19S4. Ap.J. 2ミ5. L45

Bond, J. R., \& Efstathiou. G. 105T. MNR.

Couchman. H. .I. P.. S Carlberg. R. G. 1992. ApJ. 3s9. 453

Davis, M., Si Peebles, P. J. E. 19S3. Ap.J. 26T. 465

Davis, M., Esftathiou. G.. Frent. C'. S.. L White. S. D. M. 10S5. ApJ. 292. 371

Dressler, A. 19S9, in Large Scale Structures and Peculiar Motions in The Universe, ed. David IV. Latham and L. A. Aicolaci da Costa. (Astronomical Society of the Pacific), pi9

Efstathiou, G., Sutherland. IV. J.. L Maddox. S. J. 1000. Nature. 34S. 705

Efstathiou. G., Kaiser, ‥ Saunders. W.. Lawrence. A.. Rowan-Robinson, M., Ellis, R. S., \& Frenk, C. S. 1090. MIIR.tS. 247. 10p

Geller, M. J.. \& Huchra. J. P. 19S9. Science. 246. S97

Górshi, K. M. 1991. Ap.J. 370. L5 
Groth, E. J.. S Peebles. P. J. E. 10T7. Ap.J. 217. 35.j

Hogan, C. 1991, ApJ. 369, 300

Hill, C. T., Schramm, D. ‥ \& Fry. J. X. 1080. Comments Nucl. Part. Phỵs., 19, 25

Kaiser, N. 198S. in Evolution of Large Scale Structures in the Lniverse, IAU Symposium 130, ed. J. Audouze St A. Szalay (Dordrecht: Reidel). p43

Kofman, L., Blumenthal, G. R., Hodges. H.. S Primack. J. R. 19S9, in Large Scale Structures and Peculiar Motions in the Lniverse. ed. David W. Latham and L. A. Nicolaci da Costa, (Astronomical Society of the Pacific). P339

Kofman,L.,Pogosyan, D., de Shandarin. S. 1990. M.XRAS. 242. 200

La, D., S Stcinhardt. P. J. 19S9. Phỵs. Lett.. B220. 375

Maddox, S. J.. Efstathiou. G., Sutherland. M. J.. S Loveday. J. 1990. MNRAS, $242,43 p$

Matarrese, S., Lucchin, F., Messina, A.. \& Moscardini. L. 1991b. M.NRAS, 253. 35 (.ML.M)

Messina, A., Moscardini. L.. Lucchin. F.. \& Matarrese. S. 1990. M.NRAS, 245, 244 (M.ML.M)

Moscardini, L.. Matarrese, S. Luccin. F.. L Messina. A. 1991a. M.XRAS, 24S. 424 (M.MLM)

Nusser, A., \& Dekel. A. 1990. Ap.J. 362. 14

Ostriker. J. P.. \& C'owie. L. L. 1981. ApJ. 240. L127

Peebles, P. J. E. 1950. The Large Scale Structure of the Liniverse. (Princeton: Princeton University Press).

Peebles, P. J. E. 1952. ApJ. 263. L1

Peebles, P. J. E. 10S3, ApJ. 274. 1

Salopek. D. S., Bond. J. R.. St Barcieen. J. .I. 10ミ9. Phỵs. Rer.. D40. 6

Salopek, D. S. 1991. Preprint. Ferm:1ab-p) 1 b-91 i101-.t 
Saunders, W.. Frenk. C. S.. Rowan-Robinson. .I., Efstathou. G.. Lawrence. A.. Kaiser. A., Ellis. R. S.. Crawtord. J.. Xia. I. Y.. L Pary. I. 1091, Nature. 349,32

Shanarin, S. F. 19S9, in Large-Scale Structures and Peculiar . Lotions in the Lniverse, ed. D. IV. Latham and L. A. C̈icolaci da C'osta. (Astronomical Scoiety of the Pacific). p1s9

Shandarin, S. F., \& Zel'dorich. la. B. 1059. Rev. Mod. Phỵs.. 61, 185

Smoot, G. F., Bennett, C. L., Kogut. A.. Wright, E. L.. Aymon, J., Boggess, N. W., Cheng, E. S., De Amici. G.. Gulkis, S.. Hauser, M. G., Hinshaw, G., Lineweaver, C., Loewenstein. K.. Jackson. P. D., Janssen, MI., Kaita. E., Kelsa!l. T., Keegstra. P.. Lubin. P.. Mather. J.. Merer. S. S.. Moseley, S. H., Murdock. T., Rokke. L.. Silverberg. R. F.. Tenorio. L.. Meiss. R., S Wilkinson. D. 1992, preprint

Turok, N. 19S9, Phys. Rer. Lett., 03. 26?

Weinberg. D. H., \& Cole. S. 1001, preprint.

Weinberg, D. H., S Gunn. J. E. 1090. MNRAS. 247. 260

White, S. D. M., Frenk. C'. S.. Daris. M.. \& Efstathiou. G. 1087. ApJ. 313, 505

Wise, M. B. 19S8, in The Early Lniverse. IATO ASI series. Series C. Mathematical and Physical Science: lio. 2101

Wilson, M. L.. \& Silk, J. 10S1. ApJ. 243. 14 


\section{FIGURE CAPTIONS}

Figure 1: Density distribution functions of the purely non-Gaussian fields. The horizontal axis is $y=\dot{\delta} \rho / \rho /\left(2 \sigma_{02} \sigma_{22}\right)$. The solid line is for $:=0.1$. the dotted line is for $\gamma=0.3$. the dash-dot-dot-dot line is for $\gamma=0.5$, and the long dashed line is for $\gamma=0 . S$.

Figure 2: Density distribution functions of the total density fields. The horizontai axes are $\nu=\delta \rho / \rho /\left\langle(\delta \rho / p)^{2}\right\rangle^{1 / 2}$. (a) $a=0.5 . k_{\text {eq }} / k_{c}=10$. and $B P=50 \%$. (Here $B P$ is defined the same as in the Table.1.) The solid line is for $R_{f}=$ $20 \mathrm{Mpch}^{-1}$, the dash-dot-dot-dot line is for $R_{f}=10 \mathrm{Mpch}^{-i}$. and the dotted line is for $R_{f}=2 \mathrm{Mpch}^{-1}$. (b) $a=0.5 . k_{\mathrm{eq}} / k_{r}=10$. and $R_{f}=10 \mathrm{Mpch}^{-1}$. The solid line is for the purely non-Gaussian distribution, the dash-dot-dot-dot line is for $B P=50 \%$, the dotted line is for $B P=20 \%$, the long dashed line is for Guassian distribution. (c) $R_{f}=10 \mathrm{Mpch}^{-1} \cdot B P=50 \%$. The solid line is for $a=0.5$, $k_{\text {eq }} / k_{e}=5 .$, and the dotted line is for $a=0.5$. $k_{\text {eyi }} k_{i}=10$..

Figure 3: The dots show estimates of the angular correlation function $w(\theta)$ for galaxies in the APII galaxy surrey. The dotted line is the simulation results of standard CD.M with $\Omega=1$ and $h=0.5$. The thin solid and dashed lines are the results of the linear theory for $\Omega=0.2$ scale-invariant CD.I model with $h=1$ and 0.75 , respectively. The thick solid line is the $\lambda$-body simulation results for $\Omega=0.2$ and $h=0.9$. (Reproduced from Efstathiou et al. (1990) with permission)

Figure 4: The angular correlation function $u(\theta)$. The solid line is for the standard CDM model with $\Omega=1$ and $h=0.5$. The dashed line is for $a=0.5$. $k_{\text {eq }} / k_{c}=10$., and $B P=13 \%$. The dotted line is for $a=0.5$. $k_{\text {eq }} / k_{c}=10$. and $B P=15 \%$. The dash-dot-dot-dot line is for $a=0 . \bar{j} k_{: q} / k_{c}=10$.. and $B P=20 \%$. All of the later three are consistant with the AP.M clata.

Figure 5: Power spectrum of velocity fields in units of $\left(100 \mathrm{~km} \mathrm{~s}^{-1}\right)^{2}$ without biasing. The solid line is for $a=0.5 . k_{1} / k_{c}=10$. and $B P=20 \%$. The dotted line is for the standard CDM model with $\Omega=1$, and $h=0.5$.

Figure 6: The correlation function $\left.\xi_{:}: R\right)$ of 3 -dim. velocity field in units of $\left(100 \mathrm{~km} \mathrm{~s}^{-1}\right)^{2}$. The solid line is for $a=0.5 . k_{4} / k=10$. and $B P=50 \%$. The 
dotted line is for $a=0.5 . k_{4,1} k_{-}=10 .$. a.:al $B P=20 \%$. The long dashed line is for the standard CD.I model with $\Omega=1$. and $h=0.5$.

Figure 7: The correlation function $\xi_{11}(R)$ of $\vec{\imath}(\vec{r}) \cdot \hat{r}$ in units of $\left(100 \mathrm{~km} \mathrm{~s}^{-1}\right)^{2}$. $a=0.5, k_{\text {eq }} / k_{e}=10$. The solid line is for $B P=50 \%$. The dotted line is for $B P=20 \%$. The long dashed line is for the standard CD.M model with $\Omega=1$, and $h=0.5$.

Figure 8: The rms of $\vec{i}(\vec{x}, R)=1 / 1 \int_{r i} \leq R+\vec{r}(\vec{x}+\vec{r})$ in units of $100 \mathrm{~km} \mathrm{~s}^{-1}$. $a=0.5 . k_{e y} / k_{c}=10$. The solid line is for $B P=50 \%$. The dotted line is for $B P=20 \%$. The long dashed line is for the standard CD.M model with $\Omega=1$, and $h=0.5$.

Figure 9: The rms of $i \cdot(\vec{x} . R)=1 / 1 \int_{1 \cdot \eta \leq R} d^{3} \vec{r} \vec{v}(\vec{x}+\vec{r}) \cdot \hat{r}$ in units of $100 \mathrm{~km} \mathrm{~s}^{-1}$. $a=0.5, k_{\text {eq }} / k_{c}=10$. The solid line is for $a=0.5 . k_{i j} / k=10$., and $B P=50 \%$. The dotted line is for $a=0.5 k_{. q} / k_{.}=10$. and $B P=20 ? .5$. The dash-dot-dot-dot line is for $a-0.5, k_{e y} / k_{c}=5$. and $B P=50 \%$. The long dashed line is for the standard CD.M model with $\Omega=1$. and $h=0.5$.

Figure 10: The velocity distribution function. The horizontal axis is $v=v /<$ $r^{2}>^{1 / 2}$. The solid line is for the pun ely non-Giussian velocity distribution. The long dashed line is for the relocity distribution of a Gaussian feld

Figure 11: The distribution functions of totai velocity fie!ds. The horizontal axes are $\nu=v /<l^{2}>>^{1 / 2}$. In (a) (b) and (c). the solid live is for the purely nonGaussian dist:ibution. and the long da-hed line is for the velocity distribution of a Gaussian field. (a) $a=0.5 . k_{1} / k_{0}=10$. and $B P=50 \%$. The dash-dot-dotdot line is for $R_{f}=20 \mathrm{Mpch}^{-1}$. The dotted line is for $R_{f}=10 \mathrm{Mpch}^{-1}$. The

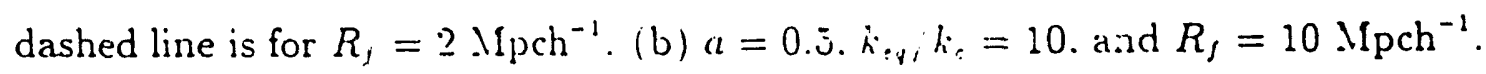
The dotted line is for $B P=50 \%$. The daslied line $i s$ for $B P=20 \%$. (c) $R_{f}=10 \mathrm{Mpch}^{-1}$, and $B P=50 \%$. The dashed line is for $a=0.5, k_{e q} / k_{c}=10$. The dotted line is for $a=0.5 . k_{\text {ey }} / k_{\circ}=5$..

Figure 12: The contributions to $\sigma^{2}\left(\theta_{r} \ldots\right.$ m.1 $)$. The dasted line is the Gaussian contribution. and the solid line is the non-Gatssian contribution. $a=0.5 \cdot k_{e q} / k_{c}=$ 10. and $B P=20 \%$. The horizontal axis is $\dot{\theta}_{F} w m:=1$. 
Figure 13: Vector plots of one slice of initial velocity fields. The areas are $128 \times$ 12S(Mpch $\left.{ }^{-1}\right)^{2}$. (a) The standard CD.M model with $\Omega=1$. and $h=0.5$. (b) The combination model with $a=0.5, k_{9,} ; k_{:}=5$. and $B P=25 \%$.

Figure 14: Vector plots of one slice of present relocity fields by using the adhesion model. The areas are $12 S \times 12 S\left(\mathrm{Mpch}^{-1}\right)^{2}$. (a) The standard CDM model with $\Omega=1$, and $h=0.5$. (b) The combination model with $a=0.5$. $k_{\text {eq }} / k_{\mathrm{s}}=5$., and $B P=25 \%$. 
TABLE 1: Parameter Regime

\begin{tabular}{ccc}
\hline \hline$k_{\text {eq }} / k_{c}$ & $a$ & $B P(\%)$ \\
\hline $5-8$ & $>0.5$ & $<20$ \\
9 & 0.5 & $17 . \overline{1}-29.3$ \\
10 & 0.5 & $13.8-22.9$ \\
11 & 0.5 & $12.4-20.5$ \\
12 & 0.5 & $11.2-18.5$ \\
13 & 0.5 & $10.2-16.9$ \\
13 & 0.4 & $13.9-23.1$ \\
14 & 0.4 & $12.5-21.3$ \\
\hline
\end{tabular}

Note.- The third column $B P$ is the non-Gaussian contribution to $\left\langle(\delta \rho / \rho)^{2}>\right.$ on $S . \mathrm{Mpch}^{-1}$ as a percentage, chosen to fit the AP.M angular correlation function $u(\theta)$.

TABLE 2: The 3-dim. rms peculiar velocity

\begin{tabular}{|c|c|c|c|c|}
\hline \multicolumn{5}{|c|}{$a=0.5 \cdot k_{e q} / k_{c}=10 . B P=15 \%$} \\
\hline & $\sigma_{v}(3.2)$ & $\sigma_{n}(15)$ & $\sigma_{2 \cdot(25)}$ & $\sigma_{v}(40)$ \\
\hline Standard CD.I & 759 & $40 \mathrm{~S}$ & 294 & $20 i$ \\
\hline Combination Model & $\pi i$ & 473 & $35 i$ & 253 \\
\hline
\end{tabular}

Note.-- The numbers in the parentheses stand for the smoothing scales in units of $\mathrm{Mpch}^{-1}$. and the rms velocity $\sigma_{r}$ is in units of $\mathrm{kms}^{-1}$. 
TABLE 3: Temperature fluctuations

\begin{tabular}{|c|c|c|}
\hline \multicolumn{3}{|c|}{$h=0.5 . \Omega=1 . a=0.5 . k_{e q} / k_{c}=10 . B P=20 \%$} \\
\hline & $(\Delta T / T)_{2}$ & $\sigma\left(\theta_{F} \| H M=10^{\circ}\right)$ \\
\hline Standard C'DXI & $5.2 \times 10^{-6}$ & $1.06 \times 10^{-5}$ \\
\hline Combination Model & $4.7 \times 10^{-6}$ & $1.01 \times 10^{-5}$ \\
\hline
\end{tabular}




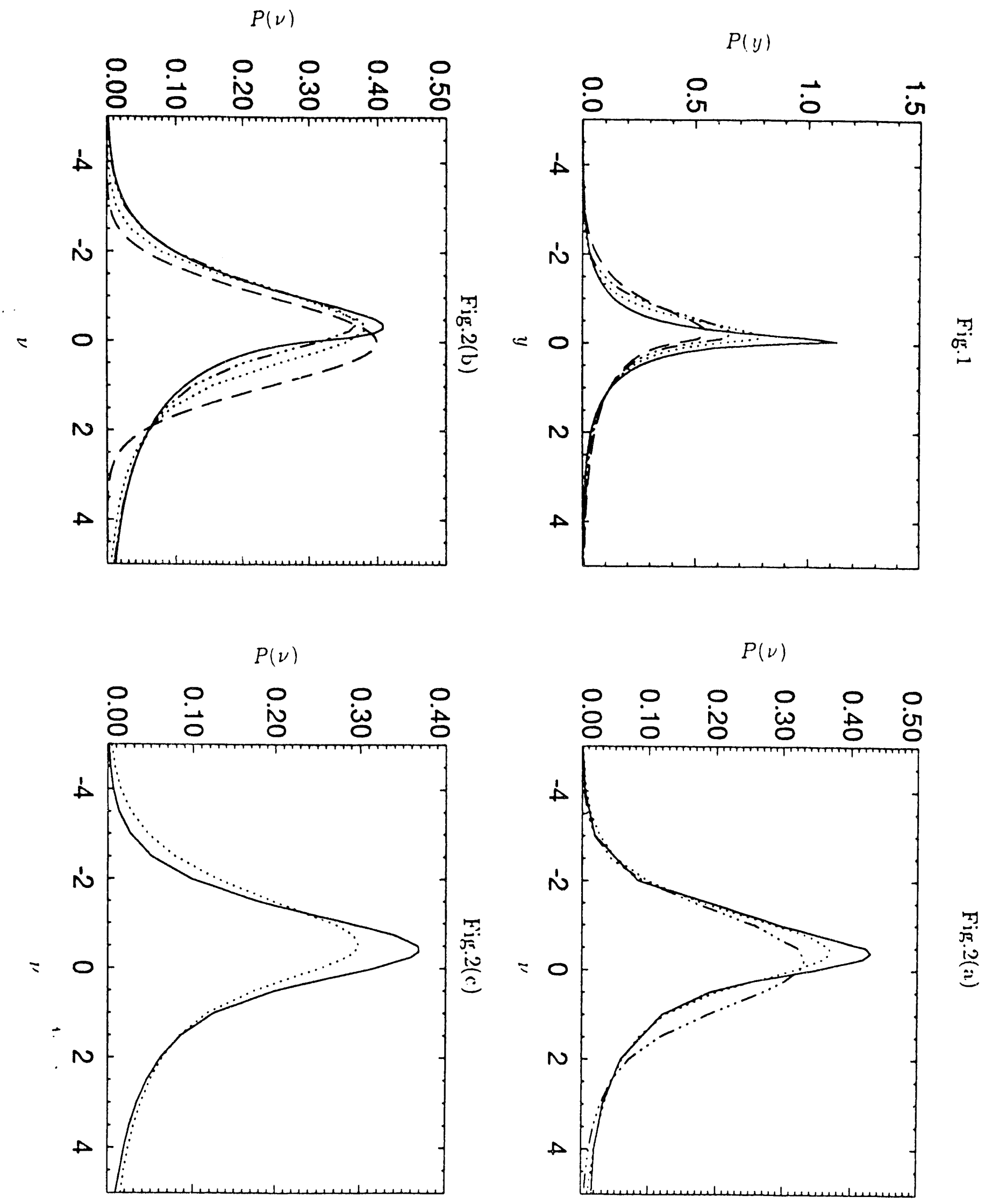


Fig.3

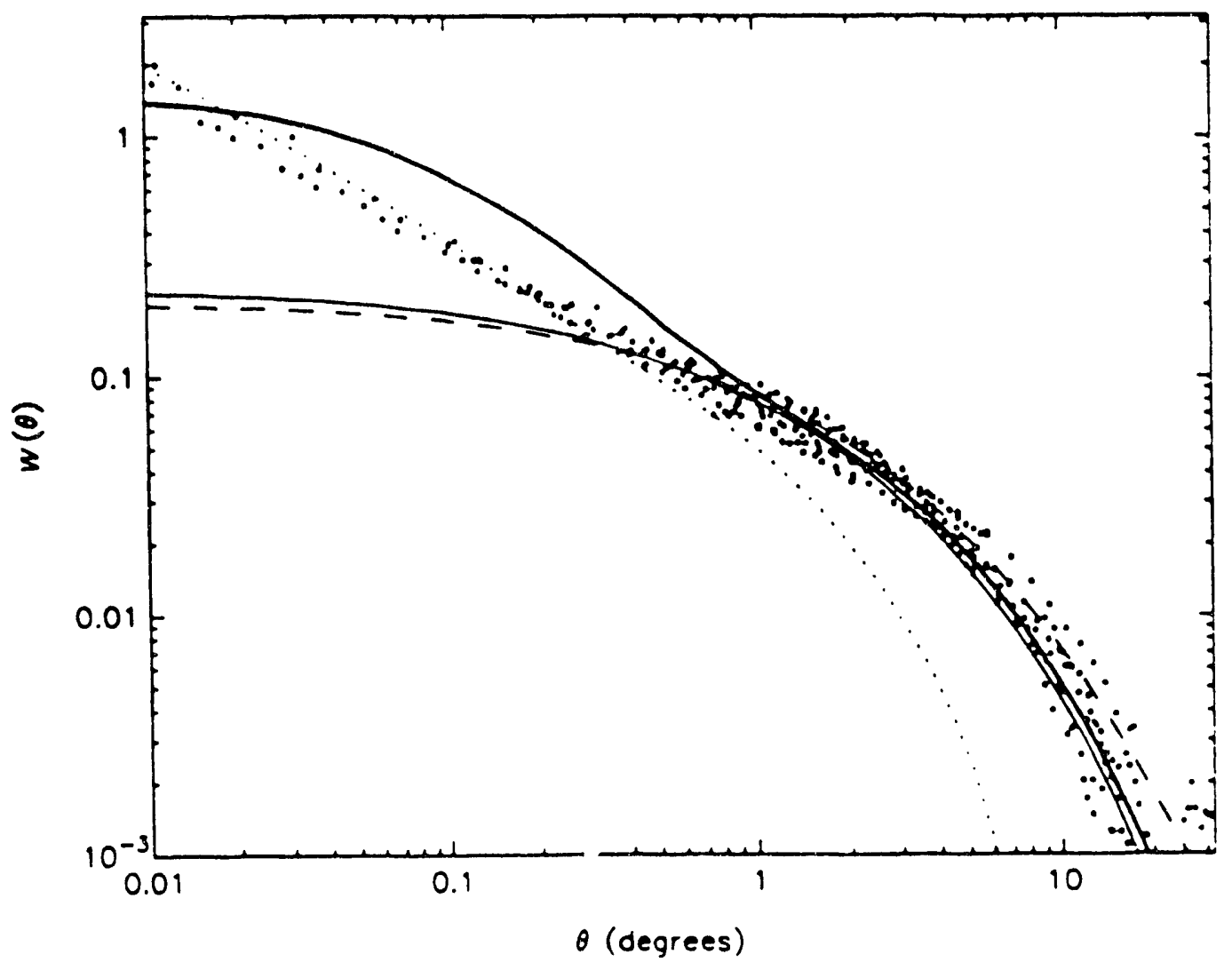


Fig.4

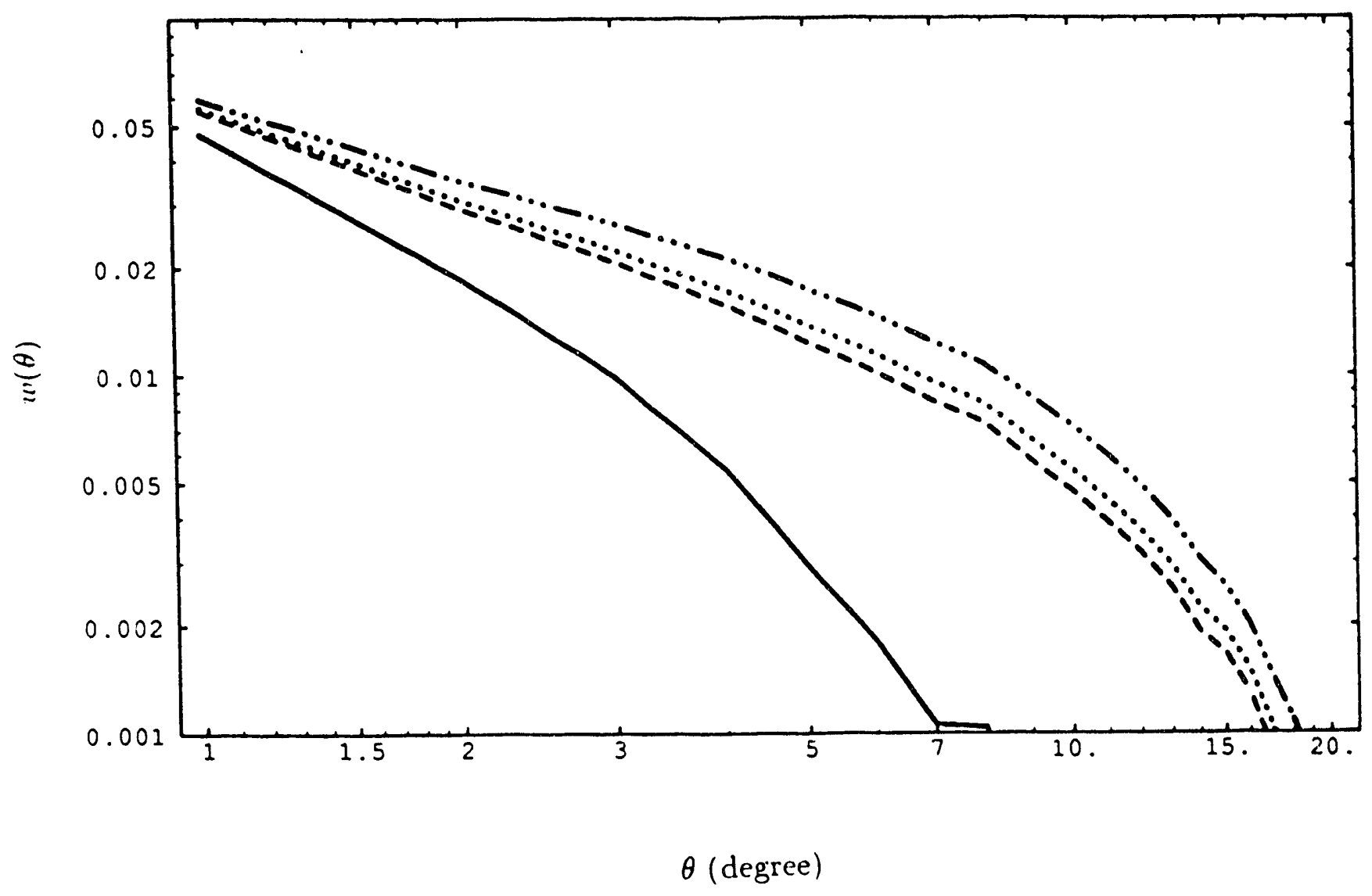


Fig.j

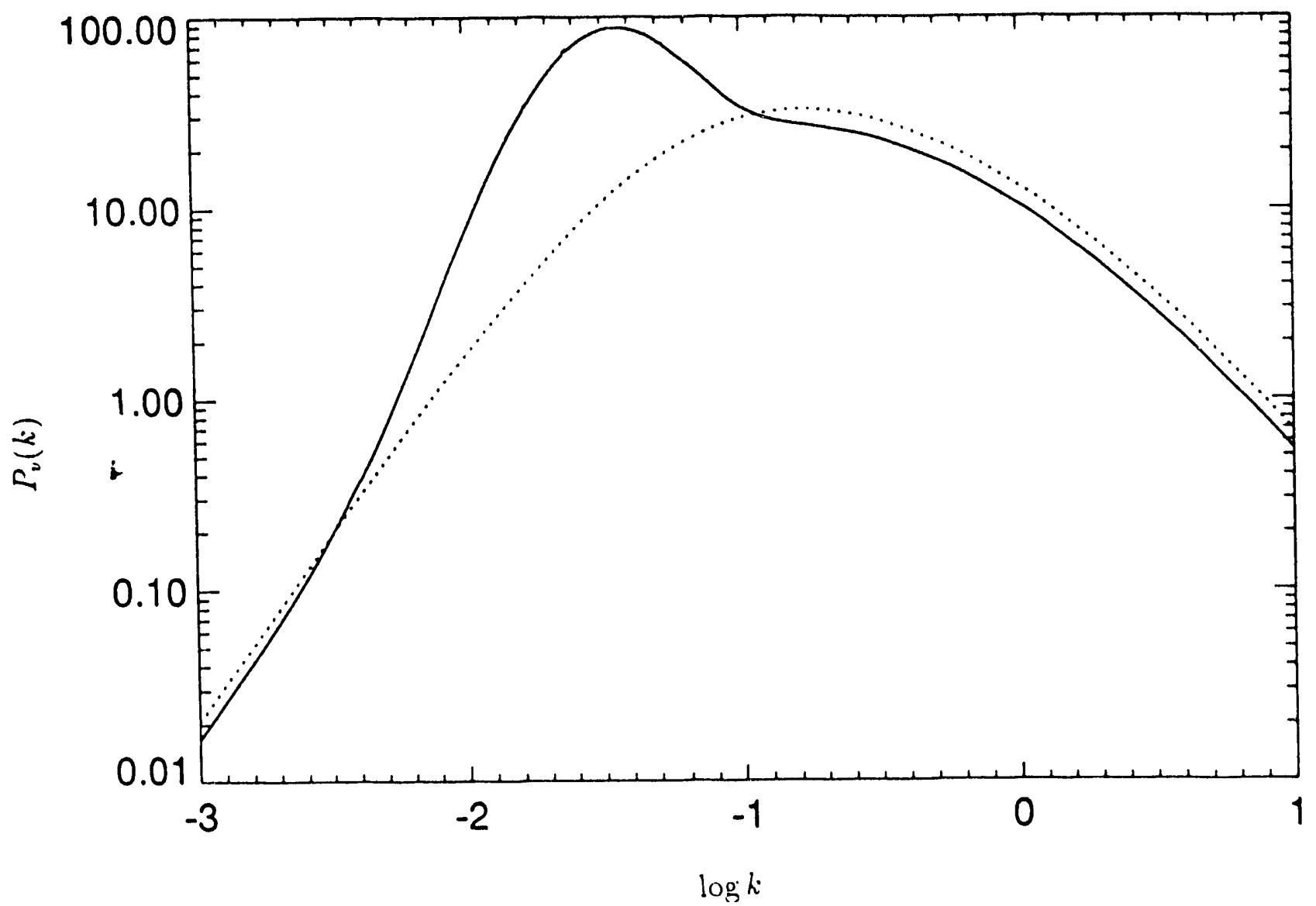



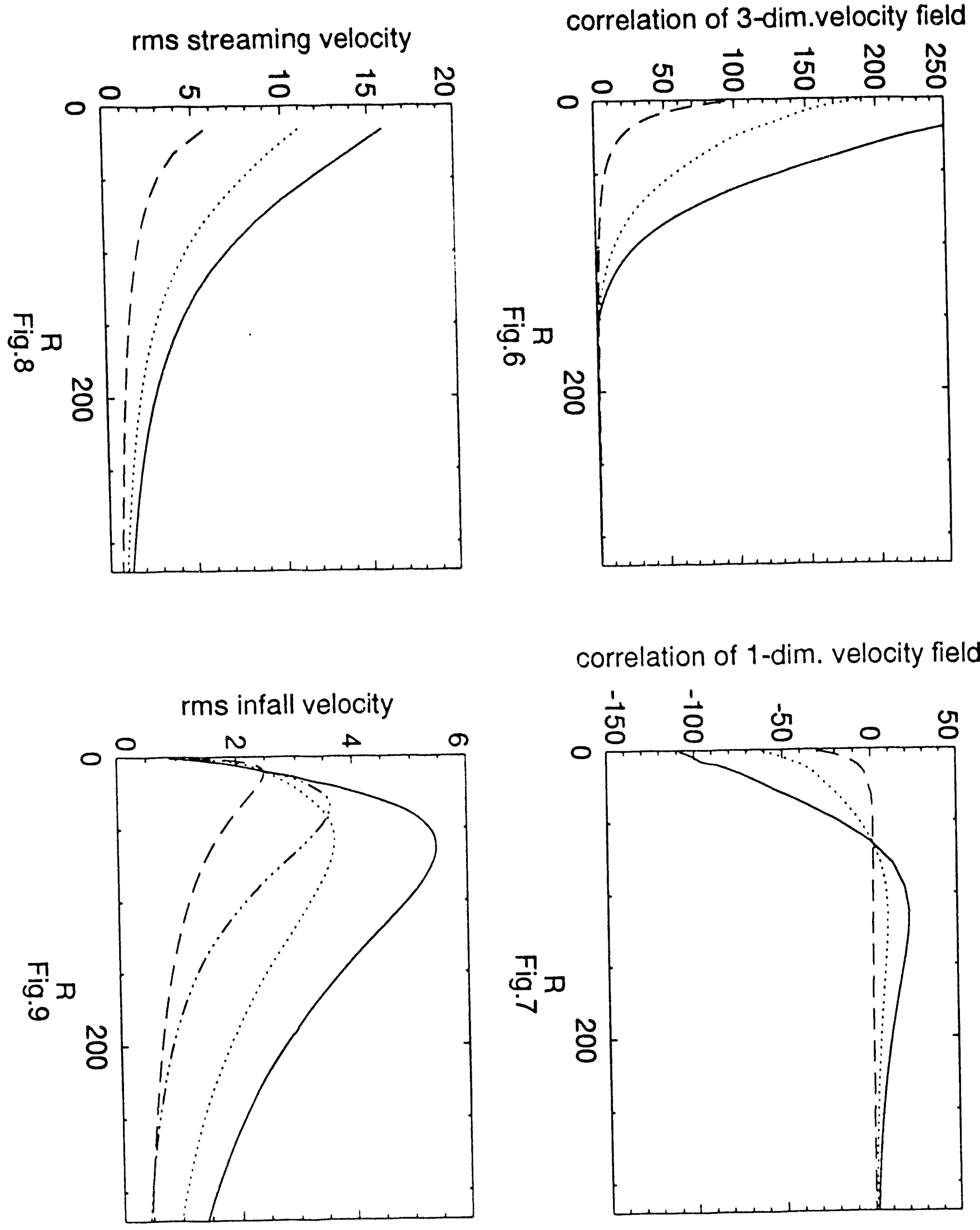


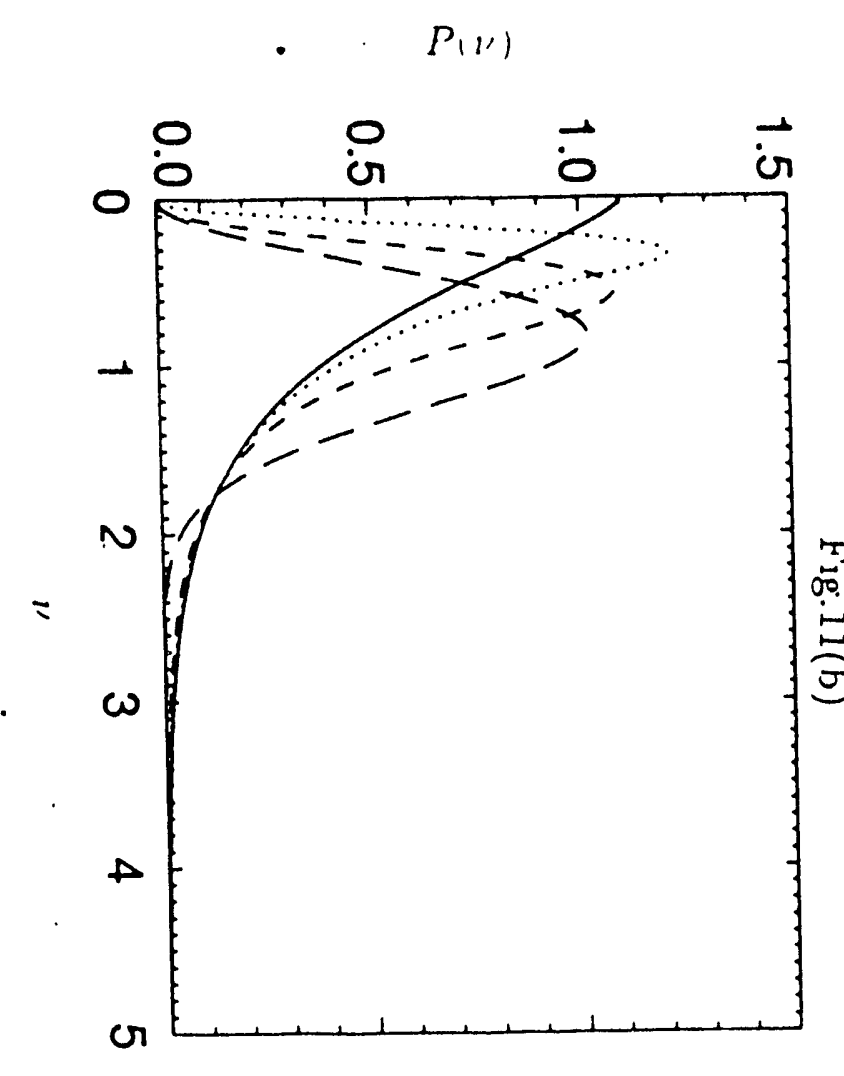

$P(\nu)$

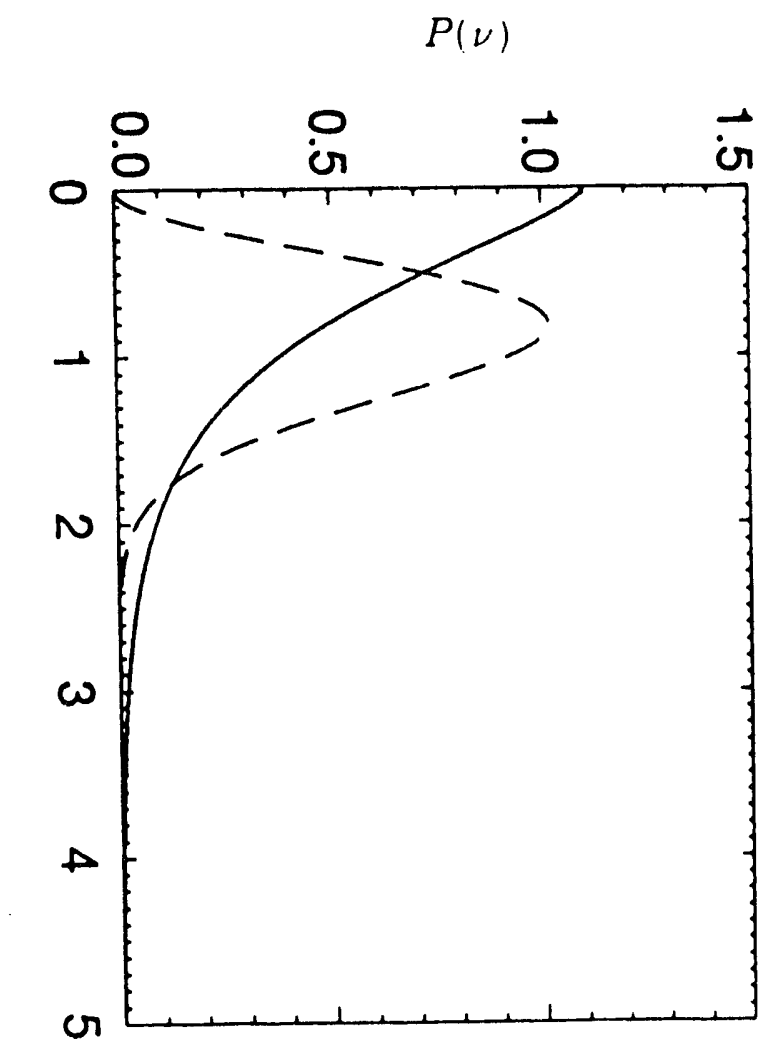

羿
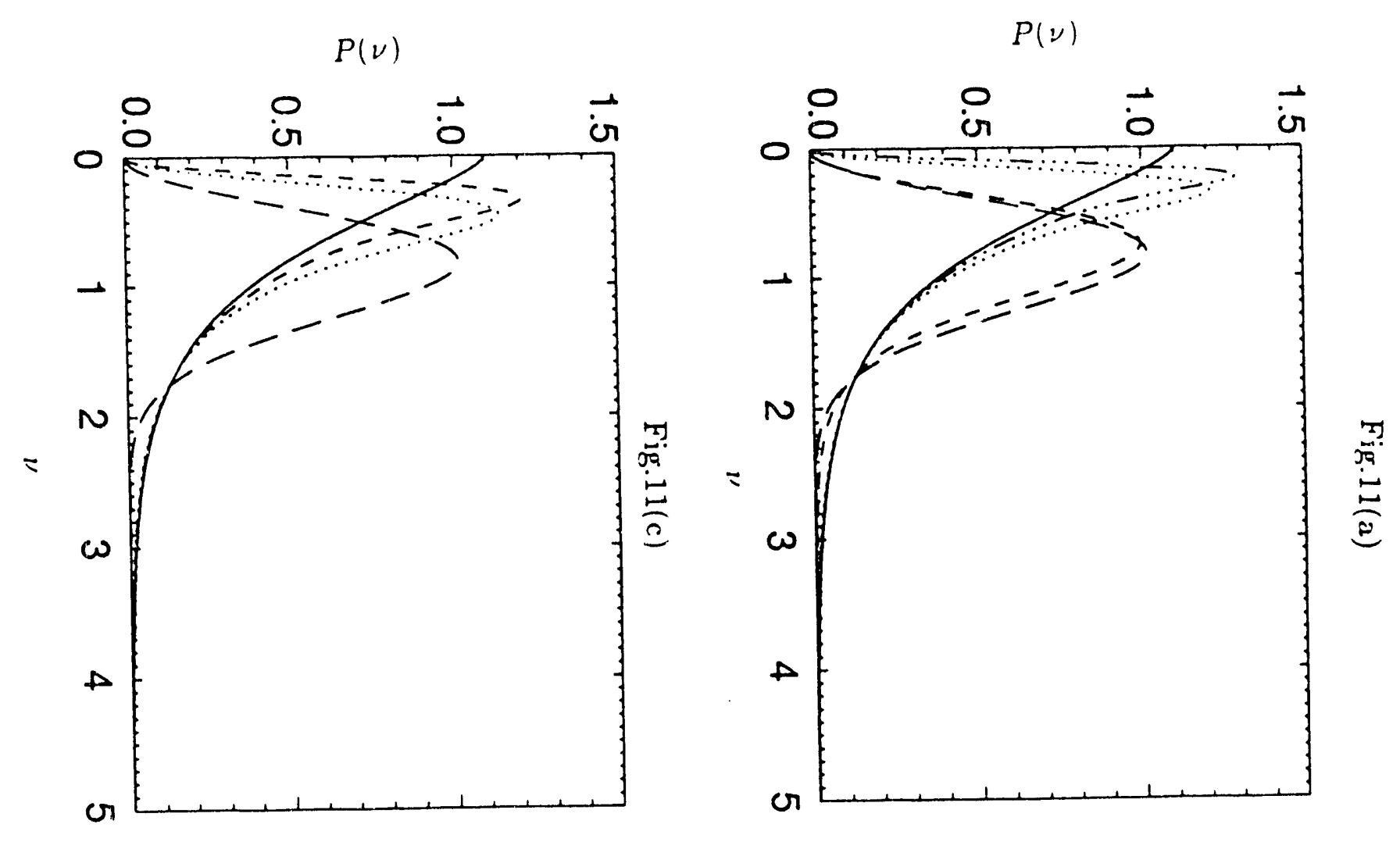
Fig.12

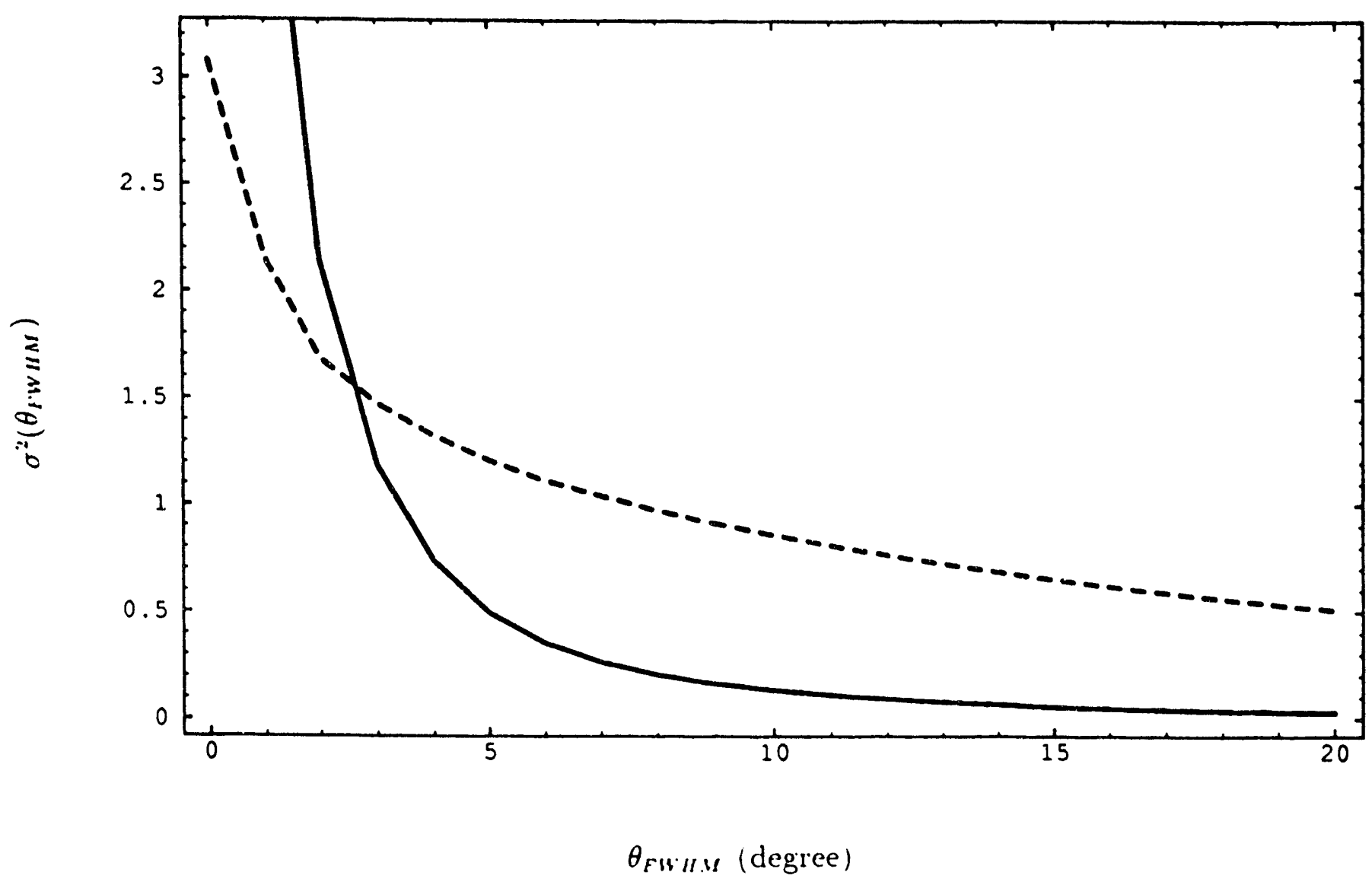


Fig.13(a)

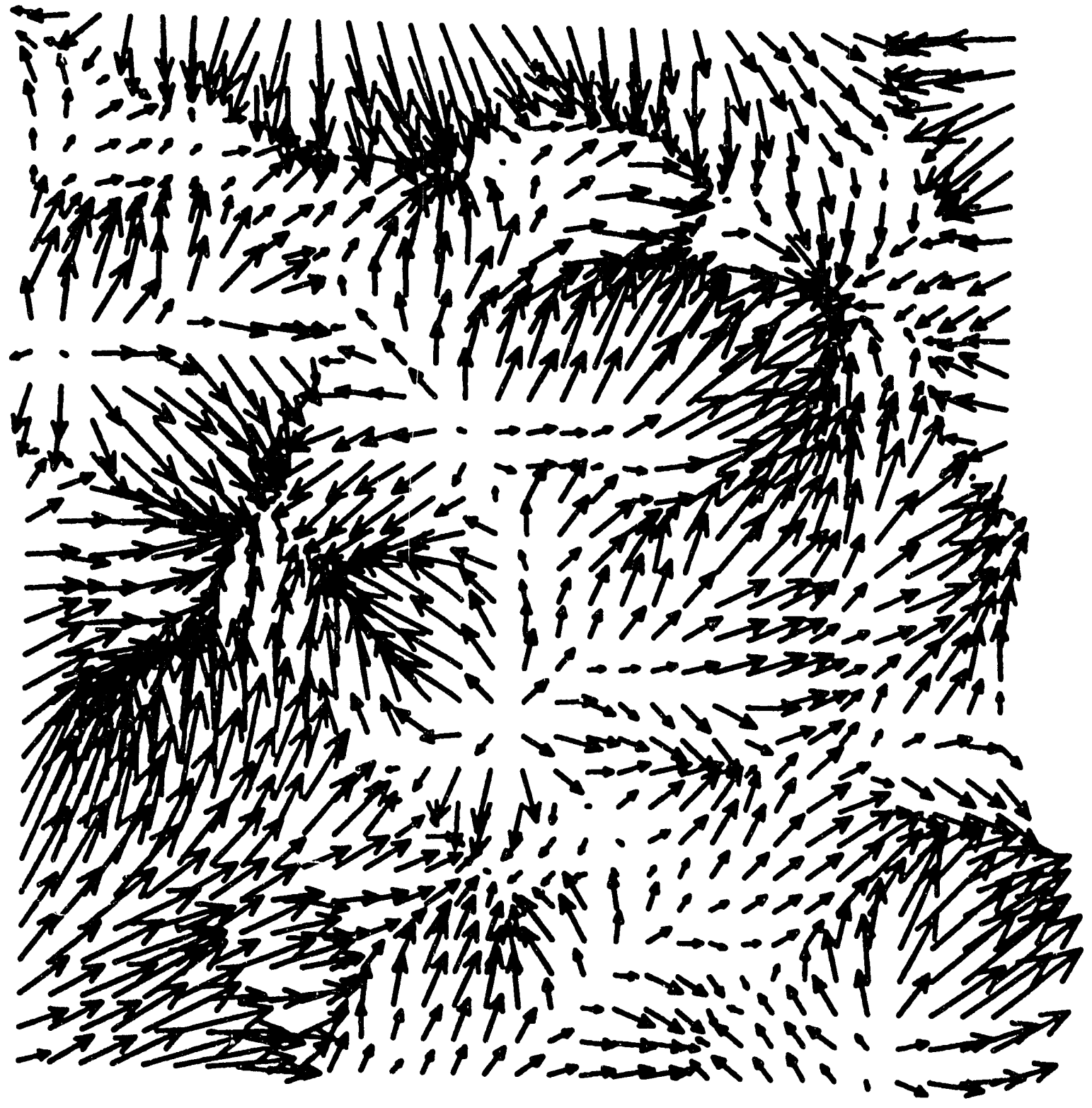


Fig.13(b)

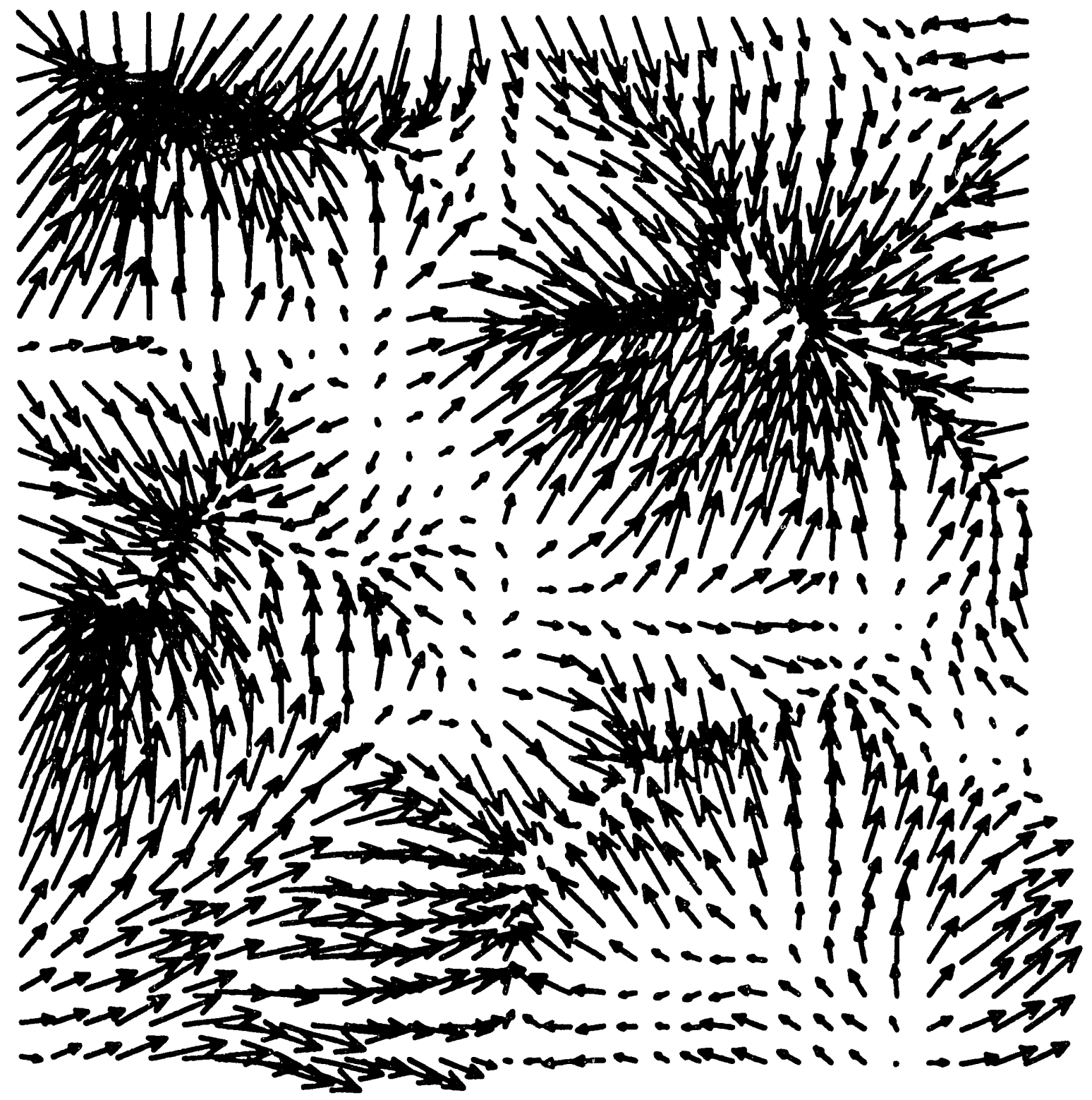


Fig.14(a)

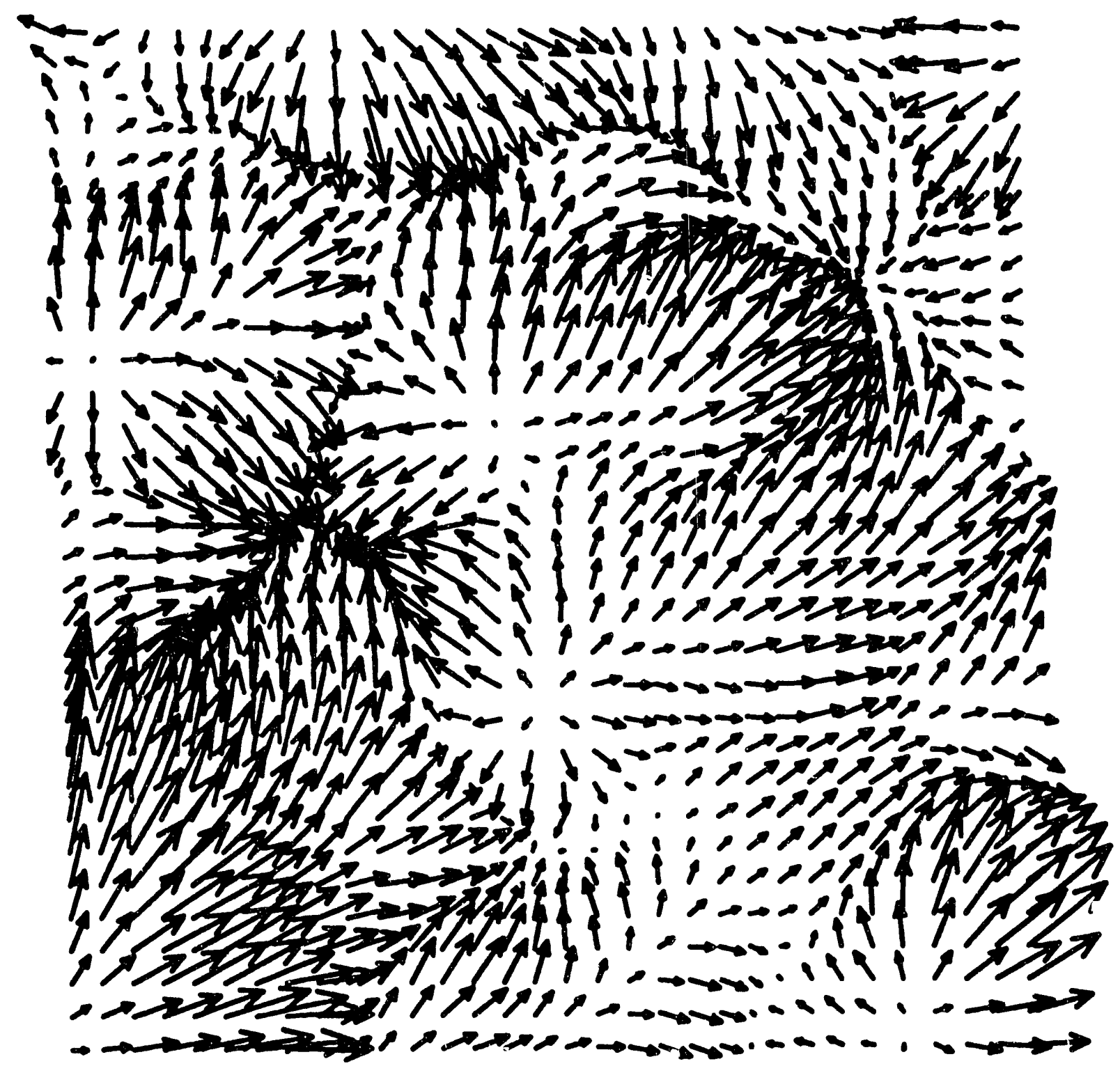


Fig. $14(b)$

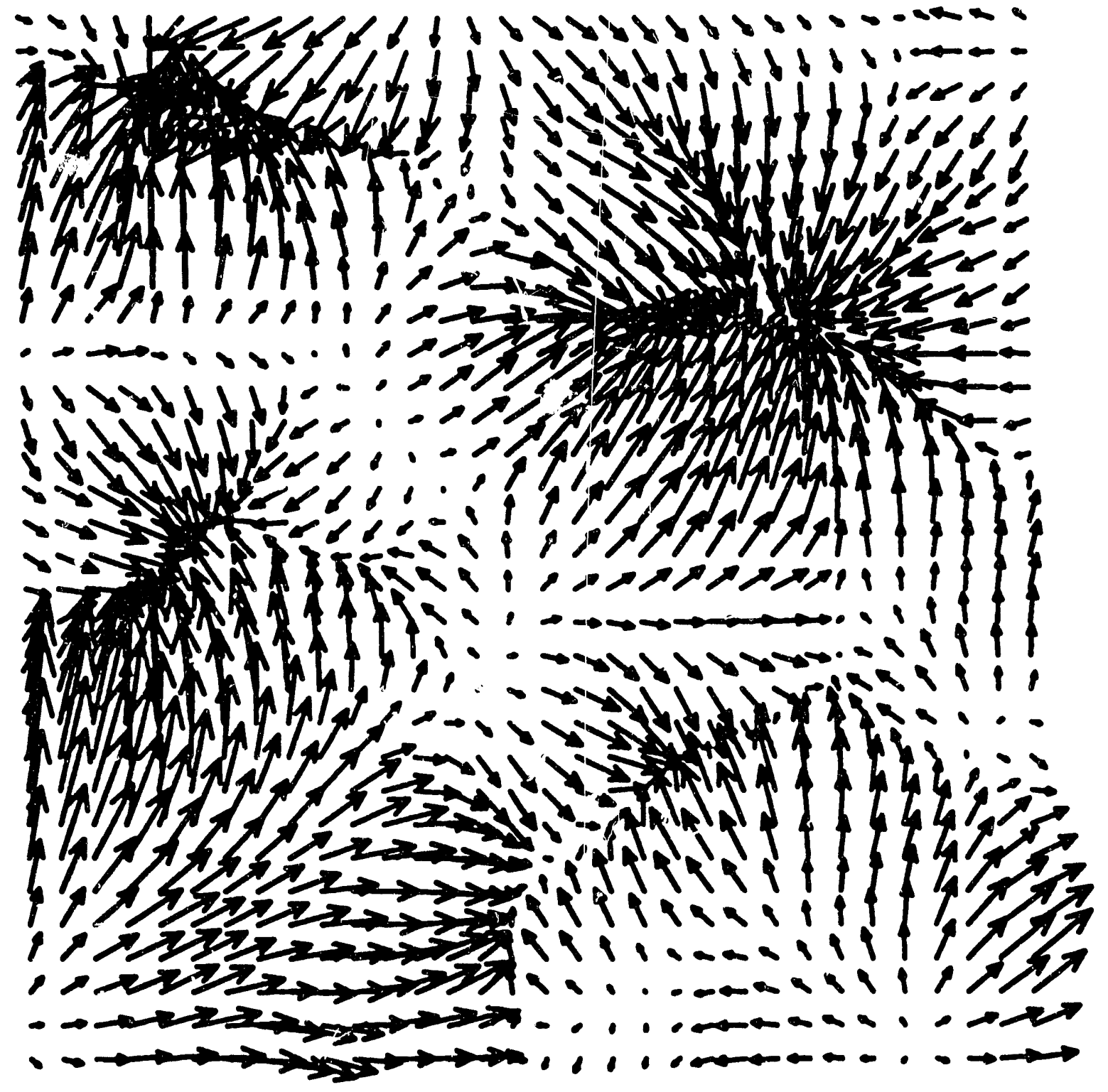



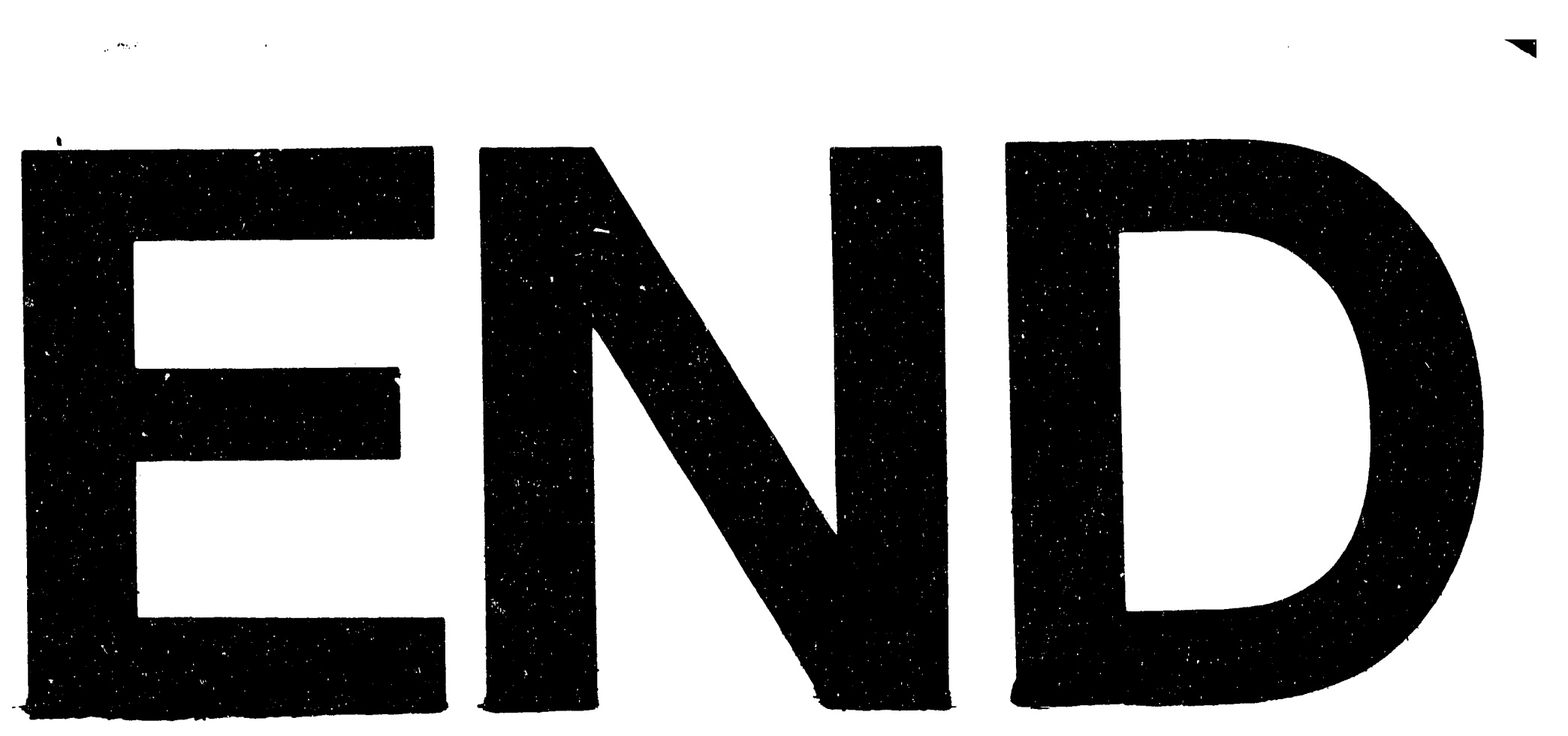

$x$
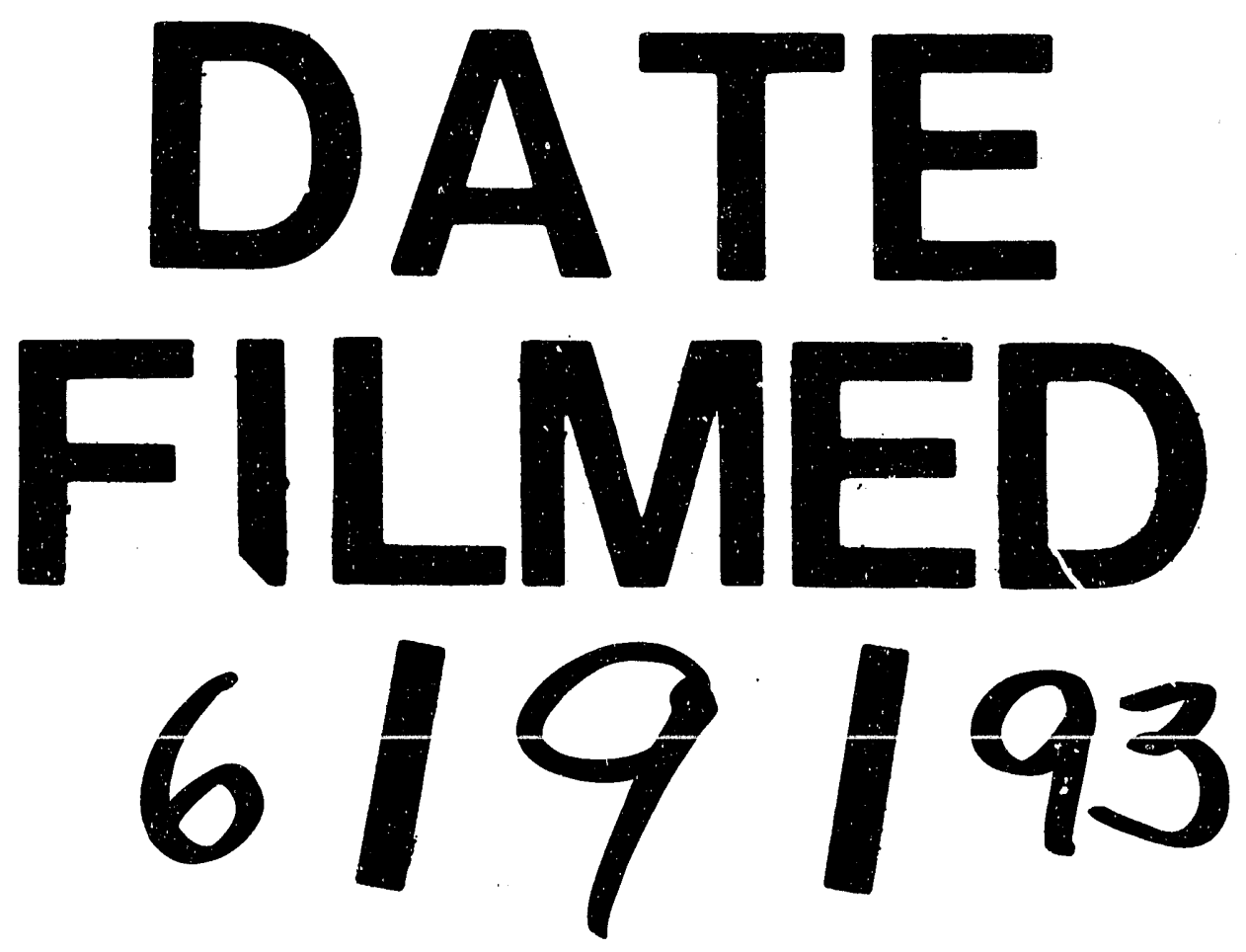
